Appl. Math. Mech. -Engl. Ed., 42(6), 819-840 (2021)

APPLIED MATHEMATICS AND MECHANICS (ENGLISH EDITION)

https://doi.org/10.1007/s10483-021-2730-7

\title{
Vibration characteristics of piezoelectric functionally graded carbon nanotube-reinforced composite doubly-curved shells*
}

\author{
V. V. THAM, H. Q. TRAN ${ }^{\dagger}, \quad$ T. M. TU \\ National University of Civil Engineering, Giai Phong Road 55, \\ Hai Ba Trung District, Hanoi 100000, Vietnam \\ (Received Dec. 6, 2020 / Revised Feb. 10, 2021)
}

\begin{abstract}
This paper presents an analytical solution for the free vibration behavior of functionally graded carbon nanotube-reinforced composite (FG-CNTRC) doubly curved shallow shells with integrated piezoelectric layers. Here, the linear distribution of electric potential across the thickness of the piezoelectric layer and five different types of carbon nanotube (CNT) distributions through the thickness direction are considered. Based on the four-variable shear deformation refined shell theory, governing equations are obtained by applying Hamilton's principle. Navier's solution for the shell panels with the simply supported boundary condition at all four edges is derived. Several numerical examples validate the accuracy of the presented solution. New parametric studies regarding the effects of different material properties, shell geometric parameters, and electrical boundary conditions on the free vibration responses of the hybrid panels are investigated and discussed in detail.
\end{abstract}

Key words free vibration, four-variable shear deformation refined theory, functionally graded carbon nanotube-reinforced composite (FG-CNTRC), piezoelectric material

Chinese Library Classification O242

2010 Mathematics Subject Classification $11 \mathrm{C} 08,30 \mathrm{G} 25,32 \mathrm{~W} 50,35 \mathrm{R} 15,65 \mathrm{~N} 12$

\section{Introduction}

Functionally graded carbon nanotube-reinforced composite (FG-CNTRC) materials are a new generation of composite materials in which carbon nanotubes (CNTs) are designed purposefully to grade with specific rules along with desired directions within an isotropic matrix. Thanks to the outstanding properties of FG-CNTRC and electromechanical properties of piezoelectric materials, the complicated FG-CNTRC structures with integrated piezoelectric layers (PFG-CNTRC) offer great potential for use in engineering, advanced aerospace, medicine, military, and automotive structural applications. The PFG-CNTRC structures are applied as smart structures to control the free vibration, suppress the forced vibration, decrease the deflection and stresses, delay the buckling, decrease the post-buckling deflection, flutter control,

* Citation: THAM, V. V., TRAN, H. Q., and TU, T. M. Vibration characteristics of piezoelectric functionally graded carbon nanotube-reinforced composite doubly-curved shells. Applied Mathematics and Mechanics (English Edition), 42(6), 819-840 (2021) https://doi.org/10.1007/s10483-021-2730-7

$\dagger$ Corresponding author, E-mail: quocth@nuce.edu.vn

Project supported by the Foundation for Science and Technology Development of National University of Civil Engineering-Ha Noi-Vietnam (No. 27-2020/KHXD-TD)

(C) The Author(s) 2021 
and suppress the snap-through phenomenon. Subsequently, researchers started to investigate the behavior of the PFG-CNTRC structures in the last few years.

Based on the three-dimensional (3D) theory of elasticity, Alibeigloo ${ }^{[1-5]}$ performed the bending and free vibration analysis of PFG-CNTRC plates and cylindrical panels (CYLs) subjected to mechanical uniform pressure, thermal load, and applied voltage field. Using a unified formulation of a finite layer model based on Reissner's mixed variational theorem, the 3D buckling responses of the PFG-CNTRC plates under bi-axial compressive loads were investigated by $\mathrm{Wu}$ and Chang ${ }^{[6]}$. In the classical lamination theory based on the Kirchhoff plate theory, the interlaminar shear deformation is neglected. Nasihatgozar et al. ${ }^{[7]}$ studied the stability of the PFG-CNTRC cylindrical panels under the axial and biaxial loadings. Applying the Ritz energy approach, the post-buckling behavior of PFG-CNTRC cylindrical shells subjected to axial compression and lateral loads in the thermal environment was investigated by Ansari et al. ${ }^{[8]}$. Using the first-order shear deformation plate theory (FSDT) with the assumption of constant transverse shear deformation, Rafiee et al. ${ }^{[9]}$ analyzed the nonlinear dynamic stability of PFG-CNTRC plates with initial geometric imperfection subjected to a combined thermal and electrical loadings and interaction of parametric and external resonance. Rafiee et al. ${ }^{[10]}$ also investigated the large amplitude free vibration of the immovable simply supported PFGCNTRC plates. Based on the FSDT and the Ritz method, Kiani ${ }^{[11]}$ developed a model for the free vibration analysis of FG-CNTRC plates with various mechanical and electrical boundary conditions. Sharma et al. ${ }^{[12]}$ implemented a finite element model with the nonlinear fuzzy logic controller to perform active vibration control of the FG-CNTRC plates. Some research papers on the analysis of PFG-CNTRC plates and shells based on the higher-order shear deformation plate theory are also available. Kolahchi et al. ${ }^{[13]}$ presented a model based on the refined piezoelasticity zig-zag theory for general wave propagation analysis of the piezoelectric sandwich plate. The free vibration behavior of the PFG-CNTRC quadrilateral spherical panel was examined by Setoodeh et al. ${ }^{[1]}$. Nguyen-Quang et al..15] introduced an extension of the isogeometric approach based on the non-uniform rational B-spline (NURBS) basis functions for the dynamic response of the laminated PFG-CNTRC plates. Based on Reddy's higher-order shear deformation theory, Selim et al. ${ }^{[16]}$ studied the impact analysis of the PFG-CNTRC plates using the element-free IMLS-Ritz model. Song et al. ${ }^{[1]}$ presented the active vibration control of FG-CNTRC plates with surface-bonded piezoelectric actuator and sensor. Selim et al. ${ }^{[18]}$ presented a novel element-free IMLS-Ritz model for the active vibration control of the FGCNTRC plate with different positions of piezoelectric sensor layer and actuator layer. Zhang et al. ${ }^{[19]}$ presented an optimal shape control of PFG-CNTRC plates with the open-loop control and the displacement feedback control gain.

Free vibration characteristics of shell structures were reported by many authors such as Tran et al. ${ }^{[20]}$, Baghlani et al. ${ }^{[21]}$ for FGM cylindrical shells; Song et al. ${ }^{[22]}$ for FG-CNTRC cylindrical shells; Zhu et al. for cylindrical nano-shell ${ }^{[23]}$, and doubly curved nano-shell ${ }^{[24]}$.

In the present work, the previous works of Tran et al. ${ }^{[25]}$ and HUU et al. ${ }^{[26}$ for PFG-CNTRC plates are utilized and developed wherein multilayer FG-CNTRC doubly curved panels (DCPs) with surface-bonded piezoelectric layers are modeled using four-variable shear deformation refined theory for the first time. By applying Navier's solution, the governing equations can be solved to obtain the vibrational responses of hybrid shell panels. Several numerical examples validate the accuracy of the present model. The effects of material properties, geometric parameters, and electrical boundary conditions on the vibrational responses of the hybrid panels are also investigated and discussed in detail.

\section{Mathematical formulation}

\subsection{Geometry and material properties}

Consider a laminated FG-CNTRC doubly curved panel (FG-CNTRC-DCP) with two perfect bonded piezoelectric layers at the top and bottom surfaces, as shown in Fig. 1. 


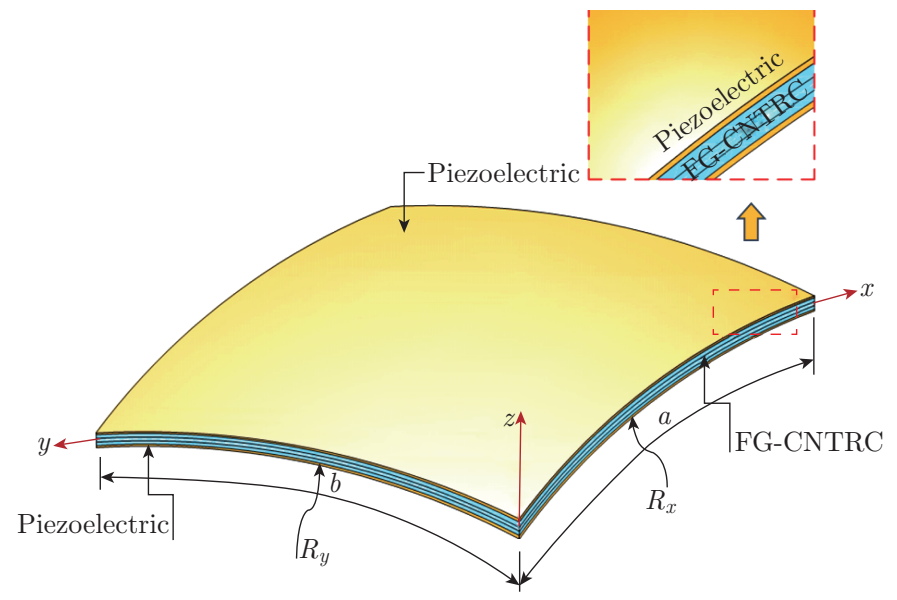

Fig. 1 Schematic of laminated PFG-CNTRC doubly curved shell panel (color online)

The panel with constant principal curvatures is referred to as an orthogonal curvilinear coordinate system $(x, y, z)$. The length, width, and two radii of principal curvatures of the middle surface of the panel are denoted by $a, b$, and $R_{x}$ and $R_{y}$, respectively. The thickness of the core and each piezoelectric layer are also marked by $h_{\mathrm{c}}$ and $h_{\mathrm{p}}$, respectively.

As shown in Fig. 2, for each layer, five types of distributions of CNT are considered. UD represents the uniform distributions, and the other four types of functionally graded distributions of CNT are denoted by FG-A, FG-V, FG-X, and FG-O.

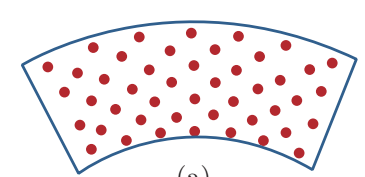

(a)

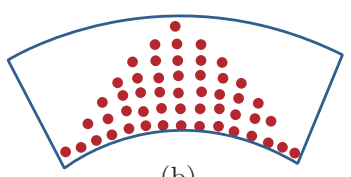

(b)

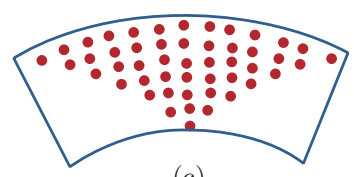

(c)

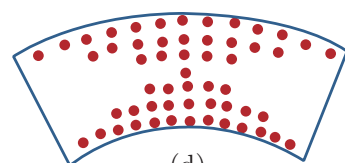

(d)

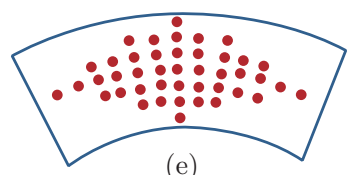

(e)

Fig. 2 Distribution types of FG-CNT reinforced composite layers: (a) UD layer; (b) FG-A layer; (c) FG-V layer; (d) FG-X layer; (e) FG-O layer (color online)

According to the distributions of CNTs, the CNT volume fractions $V_{\mathrm{CNT}}(z)$ for each FGCNTRC layer are given as ${ }^{[27]}$

$$
V_{\mathrm{CNT}}(z)= \begin{cases}V_{\mathrm{CNT}}^{*} \quad \text { for UD CNTRC, } \\ 2 V_{\mathrm{CNT}}^{*} \frac{z-z_{k}}{z_{k+1}-z_{k}} \quad \text { for FG-V CNTRC, } \\ 2 V_{\mathrm{CNT}}^{*} \frac{z_{k+1}-z}{z_{k+1}-z_{k}} \quad \text { for FG-A CNTRC, } \\ 2 V_{\mathrm{CNT}}^{*}\left(1-\frac{\left|2 z-z_{k}-z_{k+1}\right|}{z_{k+1}-z_{k}}\right) & \text { for FG-O CNTRC } \\ 2 V_{\mathrm{CNT}}^{*}\left(\frac{\left|2 z-z_{k}-z_{k+1}\right|}{z_{k+1}-z_{k}}\right) & \text { for FG-X CNTRC }\end{cases}
$$


where

$$
V_{\mathrm{CNT}}^{*}=\frac{w_{\mathrm{CNT}}}{w_{\mathrm{CNT}}+\left(\rho_{\mathrm{CNT}} / \rho_{\mathrm{m}}\right)-\left(\rho_{\mathrm{CNT}} / \rho_{\mathrm{m}}\right) w_{\mathrm{CNT}}}
$$

in which, $w_{\mathrm{CNT}}$ is the mass fraction of the $\mathrm{CNT}, \rho_{\mathrm{m}}$ and $\rho_{\mathrm{CNT}}$ are mass densities of the matrix and CNT, respectively. By using the extended rule of the mixture, the effective material properties of each FG-CNT layer can be expressed as follows ${ }^{[28]}$ :

$$
\left\{\begin{array}{l}
E_{11}(z)=\eta_{1} V_{\mathrm{CNT}}(z) E_{11}^{\mathrm{CNT}}+V_{\mathrm{m}}(z) E^{\mathrm{m}}, \quad \frac{\eta_{2}}{E_{22}(z)}=\frac{V_{\mathrm{CNT}}(z)}{E_{22}^{\mathrm{CNT}}}+\frac{V_{\mathrm{m}}(z)}{E^{\mathrm{m}}} \\
\frac{\eta_{3}}{G_{12}(z)}=\frac{V_{\mathrm{CNT}}(z)}{G_{12}^{\mathrm{CNT}}}+\frac{V_{\mathrm{m}}(z)}{G^{\mathrm{m}}}, \quad \rho(z)=V_{\mathrm{CNT}}(z) \rho^{\mathrm{CNT}}+V_{\mathrm{m}}(z) \rho^{\mathrm{m}} \\
\nu_{12}=V_{\mathrm{CNT}}^{*} \nu_{12}^{\mathrm{CNT}}+V_{\mathrm{m}}(z) \nu^{\mathrm{m}}
\end{array}\right.
$$

where $E_{11}^{\mathrm{CNT}}, E_{22}^{\mathrm{CNT}}$, and $E^{\mathrm{m}}$ are Young's moduli of CNT and matrix; $G_{12}^{\mathrm{CNT}}$ and $G^{\mathrm{m}}$ are the shear modulus of CNT and matrix; $\eta_{1}, \eta_{2}$, and $\eta_{3}$ are CNT efficiency parameters; $V_{\mathrm{m}}(z)$ and $V_{\mathrm{CNT}}(z)$ are volume fractions of matrix and CNT with the relation of $V_{\mathrm{CNT}}(z)+V_{\mathrm{m}}(z)=1$; $\nu_{12}^{\mathrm{CNT}}$ and $\nu^{\mathrm{m}}$ are Poisson's ratios of CNT and matrix.

\subsection{Approximation on mechanical displacement}

The displacements field for doubly curved shell based on the four-variable shear deformation refined theory is given by the following equations ${ }^{[29]}$ :

$$
\left\{\begin{array}{l}
u(x, y, z, t)=\left(1+\frac{z}{R_{x}}\right) u_{0}(x, y, t)-z \frac{\partial w_{\mathrm{b}}(x, y, t)}{\partial x}-f(z) \frac{\partial w_{\mathrm{s}}(x, y, t)}{\partial x} \\
v(x, y, z, t)=\left(1+\frac{z}{R_{y}}\right) v_{0}(x, y, t)-z \frac{\partial w_{\mathrm{b}}(x, y, t)}{\partial y}-f(z) \frac{\partial w_{\mathrm{s}}(x, y, t)}{\partial y} \\
w(x, y, z, t)=w_{\mathrm{b}}(x, y, t)+w_{\mathrm{s}}(x, y, t)
\end{array}\right.
$$

where $u_{0}$ and $v_{0}$ are the in-plane displacements in the directions of $x$ and $y ; w_{\mathrm{b}}$ and $w_{\mathrm{s}}$ represent the bending and shear components of the transverse displacement, respectively. The polynomial shape function is used as $f(z)=z\left(-\frac{1}{8}+\frac{3}{2}\left(\frac{z}{h}\right)^{2}\right)^{[26]}$. The strains at any point in the shell space associated with the displacement field in Eq. (4) can be obtained by applying the fundamental kinematic relations of a 3D body in curvilinear coordinates as follows ${ }^{[30]}$ :

$$
\left\{\begin{array}{l}
\varepsilon_{x}=\frac{1}{1+z / R_{x}}\left(\varepsilon_{x}^{0}+z \kappa_{x}^{\mathrm{b}}+f(z) \kappa_{x}^{\mathrm{s}}\right), \quad \varepsilon_{y}=\frac{1}{1+z / R_{y}}\left(\varepsilon_{y}^{0}+z \kappa_{y}^{\mathrm{b}}+f(z) \kappa_{y}^{\mathrm{s}}\right), \\
\gamma_{x y}=\frac{1}{1+z / R_{x}}\left(\gamma_{x y}^{0}+z \kappa_{x y}^{\mathrm{b}}+f(z) \kappa_{x y}^{\mathrm{s}}\right), \quad \gamma_{y x}=\frac{1}{1+z / R_{y}}\left(\gamma_{y x}^{0}+z \kappa_{y x}^{\mathrm{b}}+f(z) \kappa_{y x}^{\mathrm{s}}\right), \\
\gamma_{x z}=\frac{1}{1+z / R_{x}} g(z) \gamma_{x z}^{\mathrm{s}}, \quad \gamma_{y z}=\frac{1}{1+z / R_{y}} g(z) \gamma_{y z}^{\mathrm{s}},
\end{array}\right.
$$

where

$$
\left\{\begin{array}{l}
\varepsilon_{x}^{0}=\left(\frac{\partial u_{0}}{\partial x}+\frac{w_{\mathrm{b}}}{R_{x}}+\frac{w_{\mathrm{s}}}{R_{x}}\right), \quad k_{x}^{\mathrm{b}}=\left(\frac{1}{R_{x}} \frac{\partial u_{0}}{\partial x}-\frac{\partial w_{\mathrm{b}}^{2}}{\partial x^{2}}\right), \quad k_{x}^{\mathrm{s}}=-\frac{\partial^{2} w_{\mathrm{s}}}{\partial x^{2}}, \\
\varepsilon_{y}^{0}=\left(\frac{\partial v_{0}}{\partial y}+\frac{\mathrm{w}_{\mathrm{b}}}{R_{y}}+\frac{w_{\mathrm{s}}}{R_{y}}\right), \quad \kappa_{y}^{\mathrm{b}}=\left(\frac{1}{R_{y}} \frac{\partial v_{0}}{\partial y}-\frac{\partial w_{\mathrm{b}}^{2}}{\partial y^{2}}\right), \quad k_{y}^{\mathrm{s}}=-\frac{\partial^{2} w_{\mathrm{s}}}{\partial y^{2}}, \quad \gamma_{x y}^{0}=\frac{\partial v_{0}}{\partial x} \\
\kappa_{x y}^{\mathrm{b}}=\left(\frac{1}{R_{y}} \frac{\partial v_{0}}{\partial x}-\frac{\partial w_{\mathrm{b}}^{2}}{\partial x \partial y}\right), \quad \kappa_{x y}^{\mathrm{s}}=-\frac{\partial^{2} w_{\mathrm{s}}}{\partial x \partial y}, \quad \gamma_{y x}^{0}=\frac{\partial u_{0}}{\partial y} \\
\kappa_{y x}^{\mathrm{b}}=\left(\frac{1}{R_{x}} \frac{\partial u_{0}}{\partial y}-\frac{\partial w_{\mathrm{b}}^{2}}{\partial x \partial y}\right), \quad \kappa_{y x}^{\mathrm{s}}=-\frac{\partial^{2} w_{\mathrm{s}}}{\partial x \partial y}, \quad \gamma_{x z}^{\mathrm{s}}=\frac{\partial w_{\mathrm{s}}}{\partial x}, \\
\gamma_{y z}^{\mathrm{s}}=\frac{\partial w_{\mathrm{s}}}{\partial y}, \quad g(z)=\left(1-f^{\prime}(z)\right) .
\end{array}\right.
$$




\subsection{Approximation on electric potential}

In this work, the electric potential distribution in the transverse direction of each piezoelectric layer is approximated by the linear function as follows ${ }^{[31-33]}$ :

$$
\left\{\begin{array}{l}
\Phi^{\mathrm{t}}(x, y, z, t)=\left(z-\frac{h}{2}\right) \frac{1}{h_{\mathrm{p}}} \phi^{\mathrm{t}}(x, y, t)=Z_{\mathrm{p}}^{\mathrm{t}} \phi^{\mathrm{t}}(x, y, t), \quad \frac{h_{\mathrm{c}}}{2} \leqslant z \leqslant \frac{h_{\mathrm{c}}}{2}+h_{\mathrm{p}}, \\
\Phi^{\mathrm{b}}(x, y, z, t)=-\left(z+\frac{h}{2}\right) \frac{1}{h_{\mathrm{p}}} \phi^{\mathrm{b}}(x, y, t)=Z_{\mathrm{p}}^{\mathrm{b}} \phi^{\mathrm{b}}(x, y, t), \quad-\frac{h_{\mathrm{c}}}{2}-h_{\mathrm{p}} \leqslant z \leqslant-\frac{h_{\mathrm{c}}}{2} .
\end{array}\right.
$$

The electric field is related to electric potential by the following relation:

$$
E_{i}=-\nabla \Phi_{i}, \quad i=x, y, z,
$$

where $\nabla$ denotes the gradient operator. Thus, the components of the electric field are obtained as

$$
\left\{\begin{array}{l}
\left(E_{x}^{\mathrm{t}}, E_{y}^{\mathrm{t}}, E_{z}^{\mathrm{t}}\right)=-\left(\frac{1}{1+z / R_{x}} Z_{\mathrm{p}}^{\mathrm{t}} \phi_{x}^{\mathrm{t}}, \frac{1}{1+z / R_{y}} Z_{\mathrm{p}}^{\mathrm{t}} \phi_{y}^{\mathrm{t}}, \frac{1}{h_{\mathrm{p}}} \phi^{\mathrm{t}}\right) \\
\left(E_{x}^{\mathrm{b}}, E_{y}^{\mathrm{b}}, E_{z}^{\mathrm{b}}\right)=-\left(\frac{1}{1+z / R_{x}} Z_{\mathrm{p}}^{\mathrm{b}} \phi_{x}^{\mathrm{b}}, \frac{1}{1+z / R_{y}} Z_{\mathrm{p}}^{\mathrm{b}} \phi_{y}^{\mathrm{b}}, \frac{1}{h_{\mathrm{p}}} \phi^{\mathrm{b}}\right)
\end{array}\right.
$$

\subsection{Constitutive equations}

The stress-strain relation for the $k$ th CNT layer is expressed as ${ }^{[34]}$

$$
\left(\begin{array}{c}
\sigma_{x}^{k} \\
\sigma_{y}^{k} \\
\tau_{y z}^{k} \\
\tau_{x z}^{k} \\
\tau_{x y}^{k} \\
\tau_{y x}^{k}
\end{array}\right)=\left(\begin{array}{cccccc}
\bar{Q}_{11}^{k}(z) & \bar{Q}_{12}^{k}(z) & 0 & 0 & \bar{Q}_{16}^{k}(z) & \bar{Q}_{16}^{k}(z) \\
\bar{Q}_{12}^{k}(z) & \bar{Q}_{22}^{k}(z) & 0 & 0 & \bar{Q}_{26}^{k}(z) & \bar{Q}_{26}^{k}(z) \\
0 & 0 & \bar{Q}_{44}^{k}(z) & \bar{Q}_{45}^{k}(z) & 0 & 0 \\
0 & 0 & \bar{Q}_{45}^{k}(z) & \bar{Q}_{55}^{k}(z) & 0 & 0 \\
\bar{Q}_{16}^{k}(z) & \bar{Q}_{26}^{k}(z) & 0 & 0 & \bar{Q}_{66}^{k}(z) & \bar{Q}_{66}^{k}(z) \\
\bar{Q}_{16}^{k}(z) & \bar{Q}_{26}^{k}(z) & 0 & 0 & \bar{Q}_{66}^{k}(z) & \bar{Q}_{66}^{k}(z)
\end{array}\right)\left(\begin{array}{c}
\varepsilon_{x} \\
\varepsilon_{y} \\
\gamma_{y z} \\
\gamma_{x z} \\
\gamma_{x y} \\
\gamma_{y x}
\end{array}\right),
$$

in which $\bar{Q}_{i j}^{k}(z)$ are transformed material constants expressed in terms of material constants ${ }^{[35]}$,

$$
\left\{\begin{aligned}
\bar{Q}_{11}^{k}(z)= & Q_{11}^{\mathrm{c}}(z) \cos ^{4} \theta^{k}+2\left(Q_{12}^{\mathrm{c}}(z)+2 Q_{66}^{\mathrm{c}}(z)\right) \sin ^{2} \theta^{k} \cos ^{2} \theta^{k}+Q_{22}^{\mathrm{c}}(z) \sin ^{4} \theta^{k} \\
\bar{Q}_{12}^{k}(z)= & \left(Q_{11}^{\mathrm{c}}(z)+Q_{22}^{\mathrm{c}}(z)-4 Q_{66}^{\mathrm{c}}(z)\right) \sin ^{2} \theta^{k} \cos ^{2} \theta^{k}+Q_{12}^{\mathrm{c}}(z)\left(\sin ^{4} \theta^{k}+\cos ^{4} \theta^{k}\right) \\
\bar{Q}_{22}^{k}(z)= & Q_{11}^{\mathrm{c}}(z) \sin ^{4} \theta^{k}+2\left(Q_{12}^{\mathrm{c}}(z)+2 Q_{66}^{\mathrm{c}}(z)\right) \sin ^{2} \theta^{k} \cos ^{2} \theta^{k}+Q_{22}^{\mathrm{c}}(z) \cos ^{4} \theta^{k} \\
\bar{Q}_{16}^{k}(z)= & \left(Q_{11}^{\mathrm{c}}(z)-Q_{12}^{\mathrm{c}}(z)-2 Q_{66}^{\mathrm{c}}(z)\right) \sin \theta^{k} \cos ^{3} \theta^{k} \\
& +\left(Q_{12}^{\mathrm{c}}(z)-Q_{22}^{\mathrm{c}}(z)+2 Q_{66}^{\mathrm{c}}(z)\right) \sin ^{3} \theta^{k} \cos \theta^{k} \\
\bar{Q}_{26}^{k}(z)= & \left(Q_{11}^{\mathrm{c}}(z)-Q_{12}^{\mathrm{c}}(z)-2 Q_{66}^{\mathrm{c}}(z)\right) \sin ^{3} \theta^{k} \cos \theta^{k} \\
& +\left(Q_{12}^{\mathrm{c}}(z)-Q_{22}^{\mathrm{c}}(z)+2 Q_{66}^{\mathrm{c}}(z)\right) \sin \theta^{k} \cos ^{3} \theta^{k} \\
\bar{Q}_{66}^{k}(z)= & \left(Q_{11}^{\mathrm{c}}(z)+Q_{22}^{\mathrm{c}}(z)-2 Q_{12}^{\mathrm{c}}(z)-2 Q_{66}^{\mathrm{c}}(z)\right) \sin ^{2} \theta^{k} \cos ^{2} \theta^{k} \\
& +Q_{66}^{\mathrm{c}}(z)\left(\sin ^{4} \theta^{k}+\cos ^{4} \theta^{k}\right) \\
\bar{Q}_{44}^{k}(z)= & Q_{44}^{\mathrm{c}}(z) \cos ^{2} \theta^{k}+Q_{55}^{\mathrm{c}}(z) \sin ^{2} \theta^{k} \\
\bar{Q}_{45}^{k}(z)= & \left(Q_{55}^{\mathrm{c}}(z)-Q_{44}^{\mathrm{c}}(z)\right) \cos \theta^{k} \sin ^{k} \\
\bar{Q}_{55}^{k}(z)= & Q_{55}^{\mathrm{c}}(z) \cos ^{2} \theta^{k}+Q_{44}^{\mathrm{c}}(z) \sin ^{2} \theta^{k}
\end{aligned}\right.
$$

where $Q_{i j}^{\mathrm{c}}(z)$ are the plane stress-reduced stiffnesses defined in terms of the engineering constants in the material axes of the layer,

$$
\left\{\begin{array}{l}
Q_{11}^{\mathrm{c}}(z)=\frac{E_{11}(z)}{1-\nu_{12} \nu_{21}}, \quad Q_{12}^{\mathrm{c}}(z)=\frac{\nu_{12} E_{22}(z)}{1-\nu_{12} \nu_{21}}, \quad Q_{22}^{\mathrm{c}}(z)=\frac{E_{22}(z)}{1-\nu_{12} \nu_{21}}, \\
Q_{44}^{\mathrm{c}}=G_{23}(z), \quad Q_{55}^{\mathrm{c}}=G_{13}(z), \quad Q_{66}^{\mathrm{c}}=G_{12}(z) .
\end{array}\right.
$$


For the $k p$ th piezoelectric layer ( $k p=$ top, bottom), the stress components and electric displacement relations are given as ${ }^{[30,34,36-37]}$

$$
\begin{aligned}
& \left(\begin{array}{l}
\sigma_{x}^{k p} \\
\sigma_{y}^{k p} \\
\tau_{y z}^{k p} \\
\tau_{x z}^{k p} \\
\tau_{x y}^{k p} \\
\tau_{y x}^{k p}
\end{array}\right)=\left(\begin{array}{cccccc}
\bar{C}_{11}^{k p} & \bar{C}_{12}^{k p} & 0 & 0 & 0 & 0 \\
\bar{C}_{12}^{k p} & \bar{C}_{11}^{k p} & 0 & 0 & 0 & 0 \\
0 & 0 & \bar{C}_{55}^{k p} & 0 & 0 & 0 \\
0 & 0 & 0 & \bar{C}_{55}^{k p} & 0 & 0 \\
0 & 0 & 0 & 0 & \tilde{C}_{1122}^{k p} & \tilde{C}_{1122}^{k p} \\
0 & 0 & 0 & 0 & \tilde{C}_{1122}^{k p} & \tilde{C}_{1122}^{k p}
\end{array}\right)\left(\begin{array}{c}
\varepsilon_{x} \\
\varepsilon_{y} \\
\gamma_{y z} \\
\gamma_{x z} \\
\gamma_{x y} \\
\gamma_{y x}
\end{array}\right)-\left(\begin{array}{ccc}
0 & 0 & \bar{e}_{31}^{k p} \\
0 & 0 & \bar{e}_{31}^{k p} \\
0 & e_{24}^{k p} & 0 \\
e_{15}^{k p} & 0 & 0 \\
0 & 0 & 0 \\
0 & 0 & 0
\end{array}\right)\left(\begin{array}{l}
E_{x}^{k p} \\
E_{y}^{k p} \\
E_{z}^{k p}
\end{array}\right), \\
& \left(\begin{array}{l}
D_{x}^{k p} \\
D_{y}^{k p} \\
D_{z}^{k p}
\end{array}\right)=\left(\begin{array}{cccccc}
0 & 0 & 0 & e_{15}^{k p} & 0 & 0 \\
0 & 0 & e_{24}^{k p} & 0 & 0 & 0 \\
\bar{e}_{31}^{k p} & \bar{e}_{31}^{k p} & 0 & 0 & 0 & 0
\end{array}\right)\left(\begin{array}{c}
\varepsilon_{x} \\
\varepsilon_{y} \\
\gamma_{y z} \\
\gamma_{x z} \\
\gamma_{x y} \\
\gamma_{y x}
\end{array}\right)+\left(\begin{array}{ccc}
p_{11}^{k p} & 0 & 0 \\
0 & p_{11}^{k p} & 0 \\
0 & 0 & \bar{p}_{33}^{k p}
\end{array}\right)\left(\begin{array}{l}
E_{x}^{k p} \\
E_{y}^{k p} \\
E_{z}^{k p}
\end{array}\right)
\end{aligned}
$$

in which, the elastic constants for the piezoelectric layer ${ }^{[36-37]}$ are defined as

$$
\begin{cases}\bar{C}_{11}^{k p}=C_{11}^{k p}-\frac{\left(C_{13}^{k p}\right)^{2}}{C_{33}^{k p}}, & \bar{C}_{12}^{k p}=C_{12}^{k p}-\frac{\left(C_{13}^{k p}\right)^{2}}{C_{33}^{k p}}, \quad \bar{C}_{55}^{k p}=C_{55}^{k p} \\ \tilde{C}_{1122}^{k p}=\frac{\left(\bar{C}_{11}-\bar{C}_{12}\right)}{2}, & \bar{e}_{31}^{k p}=e_{31}^{k p}-\frac{C_{13}^{k p}}{C_{33}^{k p}} e_{33}^{k p}, \quad \bar{p}_{33}^{k p}=p_{33}^{k p}+\frac{\left(e_{33}^{k p}\right)^{2}}{C_{33}^{k p}}\end{cases}
$$

where $C_{i j}^{k p}$ are stiffness coefficients for the piezoelectric layers; $e_{i j}$ is the electromechanical coupling matrix; $p_{i j}$ is the dielectric permittivity matrix; $E$ is the electric field, and $D$ is the electrical displacement in the piezoelectric layer.

\subsection{Governing equation}

Hamilton's principle is applied herein to obtain the equations of motion of laminated PFGCNTRC doubly curved shell panels,

$$
\int_{0}^{t}(\delta U-\delta T) \mathrm{d} t=0
$$

where $\delta U$ is the variation of strain energy, and $\delta K$ is the variation of kinetic energy. The expression of variation of strain energy for the PFG-CNTRC panels is

$$
\begin{aligned}
\delta U= & \int_{0}^{b} \int_{0}^{a} \int_{-h / 2}^{h / 2}\left(\sigma_{x} \delta \varepsilon_{x}+\sigma_{y} \delta \varepsilon_{y}+\tau_{x y} \delta \gamma_{x y}+\tau_{y x} \delta \gamma_{y x}+\tau_{x z} \delta \gamma_{x z}+\tau_{y z} \delta \gamma_{y z}-D_{x}^{\mathrm{t}} \delta E_{x}^{\mathrm{t}}\right. \\
& \left.-D_{y}^{\mathrm{t}} \delta E_{y}^{\mathrm{t}}-D_{z}^{\mathrm{t}} \delta E_{z}^{\mathrm{t}}-D_{x}^{\mathrm{b}} \delta E_{x}^{\mathrm{b}}-D_{z}^{\mathrm{b}} \delta E_{y}^{\mathrm{b}}-D_{z}^{\mathrm{b}} \delta E_{z}^{\mathrm{b}}\right)\left(1+\frac{z}{R_{x}}\right)\left(1+\frac{z}{R_{y}}\right) \mathrm{d} z \mathrm{~d} x \mathrm{~d} y .
\end{aligned}
$$

Substituting Eq. (5), Eq. (9), Eq. (13), and Eq. (14) into Eq. (17), we can get the variation strain energy $\delta U$ as

$$
\begin{aligned}
\delta U= & \int_{0}^{b} \int_{0}^{a}\left(N_{x} \delta \varepsilon_{x}^{0}+N_{y} \delta \varepsilon_{y}^{0}+N_{x y} \delta \gamma_{x y}^{0}+N_{y x} \delta \gamma_{y x}^{0}+M_{x}^{\mathrm{b}} \delta \kappa_{x}^{\mathrm{b}}+M_{y}^{\mathrm{b}} \delta \kappa_{y}^{\mathrm{b}}+M_{x y}^{\mathrm{b}} \delta \kappa_{x y}^{\mathrm{b}}\right. \\
& +M_{y x}^{\mathrm{b}} \delta \kappa_{y x}^{\mathrm{b}}+M_{x}^{\mathrm{s}} \delta \kappa_{x}^{\mathrm{s}}+M_{y}^{\mathrm{s}} \delta \kappa_{y}^{\mathrm{s}}+M_{x y}^{\mathrm{s}} \delta \kappa_{x y}^{\mathrm{s}}+M_{y x}^{\mathrm{s}} \delta \kappa_{y x}^{\mathrm{s}}+Q_{y \mathrm{~s}} \delta \gamma_{y z}^{\mathrm{s}}+Q_{x \mathrm{~s}} \delta \gamma_{x z}^{\mathrm{s}} \\
& \left.-\bar{D}_{x}^{\mathrm{t}} \frac{\partial \delta \Phi^{\mathrm{t}}}{\partial x}-\bar{D}_{y}^{\mathrm{t}} \frac{\partial \delta \Phi^{\mathrm{t}}}{\partial y}-\bar{D}_{z}^{\mathrm{t}} \frac{\partial \delta \Phi^{\mathrm{t}}}{\partial z}-\bar{D}_{x}^{\mathrm{b}} \frac{\partial \delta \Phi^{\mathrm{b}}}{\partial x}-\bar{D}_{y}^{\mathrm{b}} \frac{\partial \delta \Phi^{\mathrm{b}}}{\partial y}-\bar{D}_{z}^{\mathrm{b}} \frac{\partial \delta \Phi^{\mathrm{b}}}{\partial z}\right) \mathrm{d} x \mathrm{~d} y
\end{aligned}
$$

where $\left(N_{x}, N_{y}, N_{x y}, N_{y x}\right),\left(M_{x}^{\mathrm{b}}, M_{y}^{\mathrm{b}}, M_{x y}^{\mathrm{b}}, M_{y x}^{\mathrm{b}}\right),\left(M_{x}^{\mathrm{s}}, M_{y}^{\mathrm{s}}, M_{x y}^{\mathrm{s}}, M_{y x}^{\mathrm{s}}\right)$, and $\left(Q_{x \mathrm{~s}}, Q_{y \mathrm{~s}}\right)$ are stress resultants given in detail in Appendix A. The variation of kinetic energy for the PFG-CNTRC panels is given as 


$$
\begin{aligned}
\delta T & =\int_{0}^{\mathrm{b}} \int_{0}^{a} \int_{-h / 2}^{h / 2} \rho(z)(\dot{u} \delta \dot{u}+\dot{v} \delta \dot{v}+\dot{w} \delta \dot{w})\left(1+\frac{z}{R_{x}}\right)\left(1+\frac{z}{R_{y}}\right) \mathrm{d} z \mathrm{~d} x \mathrm{~d} y \\
& =\delta T_{1}+\delta T_{2}+\delta T_{3}+\delta T_{4},
\end{aligned}
$$

where

$$
\begin{aligned}
\delta T_{1}= & \int_{0}^{\mathrm{b}} \int_{0}^{a}\left(\frac{1}{R_{x}}\left(-\left(\bar{I}_{1}+\frac{\bar{I}_{2}}{R_{x}}\right) \ddot{u}_{0}+\bar{I}_{2} \frac{\partial \ddot{w}_{\mathrm{b}}}{\partial x}+\bar{J}_{2} \frac{\partial \ddot{w}_{\mathrm{s}}}{\partial x}\right)\right. \\
& \left.-\bar{I}_{0} \ddot{u}_{0}-\bar{I}_{1}\left(\frac{\ddot{u}_{0}}{R_{x}}-\frac{\partial \ddot{w}_{\mathrm{b}}}{\partial x}\right)+\left(\bar{J}_{1} \frac{\partial \ddot{w}_{\mathrm{s}}}{\partial x}\right)\right) \delta u_{0} \mathrm{~d} x \mathrm{~d} y \\
\delta T_{2}= & \int_{0}^{\mathrm{b}} \int_{0}^{a}\left(\frac{1}{R_{y}}\left(-\left(\bar{I}_{1}+\frac{\bar{I}_{2}}{R_{y}}\right) \ddot{v}_{0}+\bar{I}_{2} \frac{\partial \ddot{w}_{\mathrm{b}}}{\partial y}+\bar{J}_{2} \frac{\partial \ddot{w}_{\mathrm{s}}}{\partial y}\right)\right. \\
& \left.-\bar{I}_{0} \ddot{v}_{0}-\bar{I}_{1}\left(\frac{\ddot{v}_{0}}{R_{y}}-\frac{\partial \ddot{w}_{\mathrm{b}}}{\partial y}\right)+\left(\bar{J}_{1} \frac{\partial \ddot{w}_{\mathrm{s}}}{\partial y}\right)\right) \delta v_{0} \mathrm{~d} x \mathrm{~d} y \\
\delta T_{3}= & \int_{0}^{\mathrm{b}} \int_{0}^{a}\left(-\left(\bar{I}_{1}+\frac{\bar{I}_{2}}{R_{x}}\right) \frac{\partial \ddot{\mathrm{u}}_{0}}{\partial x}+\bar{I}_{2} \frac{\partial^{2} \ddot{w}_{\mathrm{b}}}{\partial x^{2}}+\bar{J}_{2} \frac{\partial^{2} \ddot{w}_{\mathrm{s}}}{\partial x^{2}}-\left(\bar{I}_{1}+\frac{\bar{I}_{2}}{R_{y}}\right) \frac{\partial \ddot{v}_{0}}{\partial y}\right. \\
& \left.+\bar{I}_{2} \frac{\partial^{2} \ddot{w}_{\mathrm{b}}}{\partial y^{2}}+\bar{J}_{2} \frac{\partial^{2} \ddot{w}_{\mathrm{s}}}{\partial y^{2}}-\bar{I}_{0}\left(\ddot{w}_{\mathrm{b}}+\ddot{w}_{\mathrm{s}}\right)\right) \delta w_{\mathrm{b}} \mathrm{d} x \mathrm{~d} y, \\
\delta T_{4}= & \int_{0}^{\mathrm{b}} \int_{0}^{a}\left(-\left(\bar{J}_{1}+\frac{\bar{J}_{2}}{R_{x}}\right) \ddot{u}_{0}+\bar{J}_{2} \frac{\partial \ddot{w}_{\mathrm{b}}}{\partial x}+\bar{K}_{1} \frac{\partial \ddot{w}_{\mathrm{s}}}{\partial x}-\left(\bar{J}_{1}+\frac{\bar{J}_{2}}{R_{y}}\right) \ddot{v}_{0}\right. \\
& \left.+\bar{J}_{2} \frac{\partial \ddot{w}_{\mathrm{b}}}{\partial y}+\bar{K}_{1} \frac{\partial \ddot{w}_{\mathrm{s}}}{\partial y}-\bar{I}_{0}\left(\ddot{w}_{\mathrm{b}}+\ddot{w}_{\mathrm{s}}\right)\right) \delta w_{\mathrm{s}} \mathrm{d} x \mathrm{~d} y
\end{aligned}
$$

in which $\rho(z)$ is the mass density, and $\bar{I}_{i}(i=0,1,2)$ and $\bar{J}_{i}(i=1,2)$ are inertias defined by

$$
\left\{\begin{array}{l}
\bar{I}_{i}=I_{i}+I_{i+1}\left(\frac{1}{R_{x}}+\frac{1}{R_{y}}\right)+\frac{I_{i+2}}{R_{x} R_{y}} \\
\left(I_{0}, I_{1}, I_{2}, I_{3}, I_{4}\right)=\sum_{k=1}^{N} \int_{z_{k}}^{z_{+1}} \rho(z)\left(1, z, z^{2}, z^{3}, z^{4}\right) \mathrm{d} z \\
\bar{J}_{i}=f(z) \bar{I}_{i-1}, \quad \bar{K}_{1}=f^{2}(z) \bar{I}_{0} .
\end{array}\right.
$$

The governing equations can be obtained by substituting the variation of strain energy and kinetic energy from Eq. (18) and Eq. (19) into Eq. (16) and then set the coefficients of $\delta u_{0}, \delta v_{0}, \delta w_{\mathrm{b}}, \delta w_{\mathrm{s}}, \delta \varphi^{\mathrm{t}}$, and $\delta \varphi^{\mathrm{b}}$ to be zero,

$$
\left\{\begin{array}{l}
\delta u_{0}: \frac{\partial N_{x}}{\partial x}+\frac{\partial N_{y x}}{\partial y}+\frac{Q_{x \mathrm{~b}}}{R_{x}}=\bar{I}_{0} \ddot{u}_{0}+\bar{I}_{1}\left(\frac{\ddot{u}_{0}}{R_{x}}-\frac{\partial \ddot{w}_{\mathrm{b}}}{\partial x}\right)-\bar{J}_{1} \frac{\partial \ddot{w}_{\mathrm{s}}}{\partial x} \\
\delta v_{0}: \frac{\partial N_{y}}{\partial y}+\frac{\partial N_{y x}}{\partial x}+\frac{Q_{y \mathrm{~b}}}{R_{y}}=\bar{I}_{0} \ddot{v}_{0}+\bar{I}_{1}\left(\frac{\ddot{v}_{0}}{R_{y}}-\frac{\partial \ddot{w}_{\mathrm{b}}}{\partial y}\right)-\bar{J}_{1} \frac{\partial \ddot{\mathrm{w}}_{\mathrm{s}}}{\partial y} \\
\delta w_{\mathrm{b}}: \frac{N_{x}}{R_{x}}+\frac{N_{y}}{R_{y}}-\frac{\partial Q_{x \mathrm{~b}}}{\partial x}-\frac{\partial Q_{y \mathrm{~b}}}{\partial y}=-\bar{I}_{0}\left(\ddot{w}_{\mathrm{b}}+\ddot{w}_{\mathrm{s}}\right) \\
\delta w_{\mathrm{s}}: \frac{N_{x}}{R_{x}}+\frac{N_{y}}{R_{y}}-\frac{\partial \bar{Q}_{x \mathrm{~s}}}{\partial x}-\frac{\partial \bar{Q}_{y \mathrm{~s}}}{\partial y}=-\bar{I}_{0}\left(\ddot{w}_{\mathrm{b}}+\ddot{w}_{\mathrm{s}}\right) \\
\delta \varphi^{\mathrm{t}}: \frac{\partial \tilde{D}_{x}^{\mathrm{t}}}{\partial x}+\frac{\partial \tilde{D}_{y}^{\mathrm{t}}}{\partial y}+\tilde{D}_{z}^{\mathrm{t}}=0 \\
\delta \varphi^{\mathrm{b}}: \frac{\partial \tilde{D}_{x}^{\mathrm{b}}}{\partial x}+\frac{\partial \tilde{D}_{y}^{\mathrm{b}}}{\partial y}+\tilde{D}_{z}^{\mathrm{b}}=0 .
\end{array}\right.
$$


where

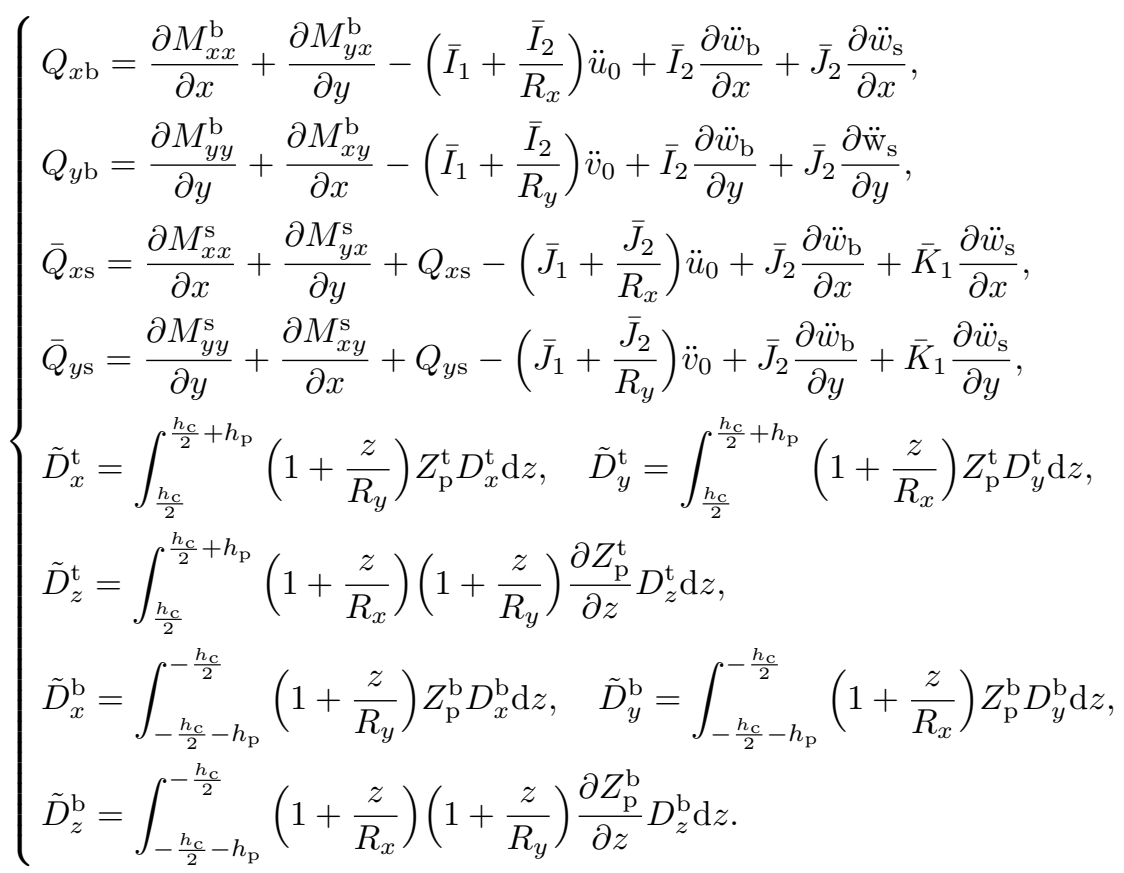

\section{Solution procedure}

In this study, two sets of simply supported boundary conditions named cross-ply (SS-1) and angle-ply (SS-2) laminates shown in Table 1 are considered.

Table 1 Two sets of simply supported boundary conditions

\begin{tabular}{cll}
\hline \multirow{2}{*}{ Edge } & \multicolumn{1}{c}{ Boundary condition } \\
\cline { 2 - 3 } & \multicolumn{1}{c}{$\mathrm{SS}-1$} & \multicolumn{1}{c}{$\mathrm{SS}-2$} \\
\hline \multirow{2}{*}{$x=0$ and $x=a$} & $v_{0}=w_{\mathrm{b}}=w_{\mathrm{s}}=w_{\mathrm{b}, y}=w_{\mathrm{s}, y}=0$ & $u_{0}=w_{\mathrm{b}}=w_{\mathrm{s}}=w_{\mathrm{b}, y}=w_{\mathrm{s}, y}=0$ \\
& $N_{x}=M_{x}^{\mathrm{b}}=M_{x}^{\mathrm{s}}=\phi^{\mathrm{t}}=\phi^{\mathrm{b}}=0$ & $N_{x y}=M_{x}^{\mathrm{b}}=M_{x}^{\mathrm{s}}=M_{x y}^{\mathrm{b}}=\phi^{\mathrm{t}}=\phi^{\mathrm{b}}=0$ \\
\multirow{2}{*}{$y=0$ and $y=b$} & $u_{0}=w_{\mathrm{b}}=w_{\mathrm{s}}=w_{\mathrm{b}, x}=w_{\mathrm{s}, x}=0$ & $v_{0}=w_{\mathrm{b}}=w_{\mathrm{s}}=w_{\mathrm{b}, x}=w_{\mathrm{s}, x}=0$ \\
& $N_{y}=M_{y}^{\mathrm{b}}=M_{y}^{\mathrm{s}}=\phi^{\mathrm{t}}=\phi^{\mathrm{b}}=0$ & $N_{y x}=M_{y}^{\mathrm{b}}=M_{y}^{\mathrm{s}}=M_{y x}^{\mathrm{b}}=\phi^{\mathrm{t}}=\phi^{\mathrm{b}}=0$ \\
\hline
\end{tabular}

Following the Navier solution procedure, the following expansion displacements for $u_{0}, v_{0}$, $w_{\mathrm{s}}, w_{\mathrm{b}}, \phi^{\mathrm{t}}$, and $\phi^{\mathrm{b}}$ are chosen to satisfy the boundary conditions given in Table 1. Here, $u_{m n}, v_{m n}, w_{m n}^{\mathrm{b}}, w_{m n}^{\mathrm{s}}, \psi_{m n}^{\mathrm{t}}$, and $\psi_{m n}^{\mathrm{b}}$ are arbitrary parameters to be determined, $\alpha=\frac{m \pi}{a}, \beta=$ $\frac{n \pi}{b}$, and $m, n$ denote the numbers of haft-waves in the $x$ - and $y$-directions, respectively.

By substituting the expansion displacement functions in Table 2 into Eq. (25), the following matrix form can be obtained:

$$
\left(\begin{array}{cccccc}
m_{11} & m_{12} & m_{13} & m_{14} & 0 & 0 \\
m_{12} & m_{22} & m_{23} & m_{24} & 0 & 0 \\
m_{13} & m_{23} & m_{33} & m_{34} & 0 & 0 \\
m_{14} & m_{24} & m_{34} & m_{44} & 0 & 0 \\
0 & 0 & 0 & 0 & 0 & 0 \\
0 & 0 & 0 & 0 & 0 & 0
\end{array}\right)\left(\begin{array}{c}
\ddot{u}_{m n} \\
\ddot{v}_{m n} \\
\ddot{w}_{m n}^{\mathrm{b}} \\
\ddot{w}_{m n}^{\mathrm{s}} \\
\ddot{\psi}_{m n}^{\mathrm{t}} \\
\ddot{\psi}_{m n}^{\mathrm{b}}
\end{array}\right)
$$




$$
+\left(\begin{array}{llllll}
k_{11} & k_{12} & k_{13} & k_{14} & k_{15} & k_{16} \\
k_{12} & k_{22} & k_{23} & k_{24} & k_{25} & k_{26} \\
k_{13} & k_{23} & k_{33} & k_{34} & k_{35} & k_{36} \\
k_{14} & k_{24} & k_{34} & k_{44} & k_{45} & k_{46} \\
k_{15} & k_{25} & k_{35} & k_{45} & k_{55} & k_{56} \\
k_{16} & k_{26} & k_{36} & k_{46} & k_{56} & k_{66}
\end{array}\right)\left(\begin{array}{c}
u_{m n} \\
v_{m n} \\
w_{m n}^{\mathrm{b}} \\
w_{m n}^{\mathrm{s}} \\
\psi_{m n}^{\mathrm{t}} \\
\psi_{m n}^{\mathrm{b}}
\end{array}\right)=\left(\begin{array}{l}
0 \\
0 \\
0 \\
0 \\
0 \\
0
\end{array}\right),
$$

where $m_{i j}$ and $k_{i j}$ are given in Appendices B1 and B2 for SS-1 and SS-2 boundary conditions, respectively. Equation (27) can be rewritten in the short form as

$$
\left(\begin{array}{cc}
M_{u u} & 0 \\
0 & 0
\end{array}\right)\left(\begin{array}{l}
\ddot{u} \\
\ddot{\phi}
\end{array}\right)+\left(\begin{array}{ll}
K_{u u} & K_{u \phi} \\
K_{\phi u} & K_{\phi \phi}
\end{array}\right)\left(\begin{array}{l}
u \\
\phi
\end{array}\right)=\left(\begin{array}{l}
0 \\
0
\end{array}\right),
$$

Table 2 Expansion displacements $\left(u_{0}, v_{0}, w_{\mathrm{s}}, w_{\mathrm{b}}, \phi^{\mathrm{t}}, \phi^{\mathrm{b}}\right)$

\begin{tabular}{ccc}
\hline Displacement & \multicolumn{1}{c}{ Boundary condition } \\
\cline { 2 - 3 }$u_{0}(x, y, t)$ & $\sum_{m=1}^{\infty} \sum_{n=1}^{\infty} u_{m n} \cos (\alpha x) \sin (\beta y)$ & $\mathrm{SS}-2$ \\
$v_{0}(x, y, t)$ & $\sum_{m=1}^{\infty} \sum_{n=1}^{\infty} u_{m n} \sin (\alpha x) \cos (\beta y)$ \\
$w_{\mathrm{b}}(x, y, t)$ & $\sum_{m=1}^{\infty} v_{m n} \sin (\alpha x) \cos (\beta y)$ & $\sum_{m=1}^{\infty} v_{m n} \cos (\alpha x) \sin (\beta y)$ \\
$w_{\mathrm{s}}(x, y, t)$ & $\sum_{m=1}^{\infty} \sum_{n=1}^{\infty} w_{m n}^{\mathrm{b}} \sin (\alpha x) \sin \beta y$ & $\sum_{m=1}^{\infty} \sum_{n=1}^{\infty} w_{m n}^{\mathrm{b}} \sin (\alpha x) \sin (\beta y)$ \\
$\phi^{\mathrm{t}}(x, y, t)$ & $\sum_{m=1}^{\infty} \sum_{n=1}^{\infty} w_{m n}^{\mathrm{s}} \sin (\alpha x) \sin \beta y$ & $\sum_{m=1}^{\infty} \sum_{n=1}^{\infty} w_{m n}^{\mathrm{s}} \sin (\alpha x) \sin (\beta y)$ \\
$\phi^{\mathrm{b}}(x, y, t)$ & $\sum_{m=1}^{\infty} \sum_{n=1}^{\infty} \psi_{m n}^{\mathrm{t}} \sin (\alpha x) \sin \beta y$ & $\sum_{m=1}^{\infty} \sum_{n=1}^{\infty} \psi_{m n}^{\mathrm{t}} \sin (\alpha x) \sin (\beta y)$ \\
\hline
\end{tabular}

where $M_{u u}$ is the mass matrix, $K_{u u}$ is the elastic matrix, $K_{u \phi}$ is the piezoelectric matrix, and $K_{\phi \phi}$ is the permittivity matrix. Substituting the solution of the second equation into the first equation in Eq. (24), we obtain a short form of the equation for vibration characteristics of FG-CNTRC shell panels integrated with piezoelectric layers,

$$
\left(M_{u u}\right)(\ddot{u})+\left(\left(K_{u u}\right)-\left(K_{u \phi}\right)\left(K_{\phi \phi}\right)^{-1}\left(K_{\phi u}\right)\right)(u)=0 .
$$

For free vibration, Eq. (29) reduces to an eigenvalue problem by setting $(u)=(\hat{u}) \mathrm{e}^{\mathrm{i} \omega t}$

$$
\left(\left(K_{u u}\right)-\left(K_{u \phi}\right)\left(K_{\phi \phi}\right)^{-1}\left(K_{\phi u}\right)-\omega^{2}\left(M_{u u}\right)\right)(\hat{u})=0 .
$$

Equation (30) is associated with the natural frequencies of an open-circuit (Opc) PFG-CNTRC doubly curved shell panels (see Fig. 3(a)). For the closed-circuit (Clc) (see Fig. 3(b)) condition, both the upper and lower piezoelectric layers are grounded cause the electric displacement disappears from Eq. (28). Hence, we obtain the following eigenvalue problem for the Clc boundary condition:

$$
\left(\left(K_{u u}\right)-\omega^{2}\left(M_{u u}\right)\right)(\hat{u})=0 .
$$

The eigensolution of free vibration for PFG-CNTRC-DCP can be obtained using a general eigenvalue approach. 


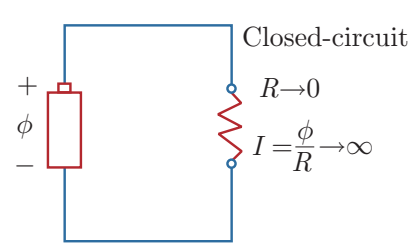

(a)

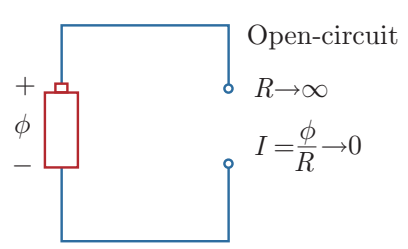

(b)

Fig. 3 Electrical boundary conditions: (a) closed-circuit and (b) open-circuit

\section{Numerical results and discussion}

In this section, several comparison studies are conducted to verify the accuracy of instant solution in predicting natural frequencies of a simply supported isotropic doubly curved shell panel and a rectangular FG-CNTRC; both of these structures are integrated with piezoelectric layers at the top and bottom surfaces. Furthermore, the effects of involved parameters such as CNT volume fractions, distribution types of CNT, and different geometric parameters on free vibration response of PFG-CNTRC doubly curved shell panel are also investigated in detail. Unless mentioned otherwise, the material properties of PFG-CNTRC doubly curved shell panels are given in Table 3 .

Table 3 Material properties of piezoelectric and FG-CNT materials

\begin{tabular}{|c|c|c|c|c|c|}
\hline \multirow{2}{*}{ Property } & \multicolumn{3}{|c|}{ Substrate layer } & \multicolumn{2}{|c|}{ Piezoelectric layer } \\
\hline & $\operatorname{PmPV}^{[15]}$ & $\mathrm{CNT}^{[15]}$ & $\mathrm{Al}_{2} \mathrm{O}_{3}[32]$ & $\mathrm{PZT}-4^{[32]}$ & PZT-5A ${ }^{[15]}$ \\
\hline$\rho /\left(\mathrm{kg} \cdot \mathrm{m}^{-3}\right)$ & 1150 & 1400 & 3800 & 7500 & 7750 \\
\hline$E_{11} / \mathrm{GPa}$ & $E^{\mathrm{m}}=2.5$ & $5.64 \times 10^{3}$ & 380 & - & $E=63$ \\
\hline$E_{22} / \mathrm{GPa}$ & $E^{\mathrm{m}}=2.5$ & $7.08 \times 10^{3}$ & 380 & - & - \\
\hline$G_{12} / \mathrm{GPa}$ & - & 1.9455 & & - & $G=23.3$ \\
\hline$\nu_{12}$ & 0.34 & 0.175 & 0.3 & - & 0.35 \\
\hline$C_{11} / \mathrm{GPa}$ & - & - & - & 139 & - \\
\hline$C_{12} / \mathrm{GPa}$ & - & - & - & 77.8 & - \\
\hline$C_{13} / \mathrm{GPa}$ & - & - & - & 74.3 & - \\
\hline$C_{33} / \mathrm{GPa}$ & - & - & - & 115 & - \\
\hline$C_{44,55} / \mathrm{GPa}$ & - & - & - & 25.6 & - \\
\hline$C_{66} / \mathrm{GPa}$ & - & - & - & 30.6 & - \\
\hline$e_{31} / \mathrm{cm}^{-2}$ & - & - & - & -5.2 & -7.209 \\
\hline$e_{33} / \mathrm{cm}^{-2}$ & - & - & - & 15.1 & 15.12 \\
\hline$e_{15} / \mathrm{cm}^{-2}$ & - & - & - & 12.7 & 12.322 \\
\hline$p_{11} /\left(\mathrm{nFm}^{-1}\right)$ & - & - & - & 6.75 & 1.53 \\
\hline$p_{33} /\left(\mathrm{nFm}^{-1}\right)$ & - & - & - & 5.9 & 1.50 \\
\hline
\end{tabular}

Also, the CNT efficiency parameters $\eta_{j}(j=1,2,3)$ associated with a given volume fraction $V_{\mathrm{CNT}}^{*} \operatorname{are}^{[15]}: \eta_{1}=0.137, \eta_{2}=1.022$ for the case of $V_{\mathrm{CNT}}^{*}=0.12, \eta_{1}=0.142, \eta_{2}=1.626$ for the case of $V_{\mathrm{CNT}}^{*}=0.17$, and $\eta_{1}=0.141, \eta_{2}=1.585$ for the case of $V_{\mathrm{CNT}}^{*}=0.28$, and in all cases $\eta_{3}=0.7 \eta_{2}$.

\subsection{Comparison studies}

Since there are no published results for the PFG-CNTRC doubly curved shell in the open literature, two numerical examples predict the free vibration behaviors of the piezoelectric isotropic doubly curved shell panels, and the PFG-CNTRC plates are performed to validate the present approach.

\subsubsection{Example 1}

Firstly, the fundamental frequencies of the simply supported isotropic doubly curved shell panel with surface-bonded piezoelectric layers for different geometric parameters are presented in Table 4. The Opc and Clc of electric boundary conditions are also considered. The shell 
Table 4 Fundamental frequencies $\omega$ of piezoelectric isotropic DCP $\left(a / b=1, R_{y} / b=5\right)$

\begin{tabular}{|c|c|c|c|c|c|c|c|c|}
\hline \multirow{2}{*}{$a / R_{x}$} & \multirow{2}{*}{$h / a$} & \multirow{2}{*}{$h_{\mathrm{p}} / h$} & \multicolumn{3}{|c|}{$\omega_{\mathrm{Clc}} / \mathrm{Hz}$} & \multicolumn{3}{|c|}{$\omega_{\mathrm{Opc}} / \mathrm{Hz}$} \\
\hline & & & Present & Ref. [38] & Ref. [32] & Present & Ref. [38] & Ref. [32] \\
\hline \multirow[t]{4}{*}{-0.2} & 0.1 & 0.1 & 823.126 & 824.049 & 823.997 & 840.081 & 841.189 & 843.029 \\
\hline & & 0.2 & 787.613 & 788.430 & 788.091 & 819.929 & 820.477 & 826.775 \\
\hline & 0.2 & 0.1 & 1484.893 & 1483.998 & 1483.888 & 1514.076 & 1512.112 & 1515.550 \\
\hline & & 0.2 & 1400.806 & 1398.792 & 1398.093 & 1457.032 & 1449.614 & 1461.337 \\
\hline \multirow[t]{4}{*}{0} & 0.1 & 0.1 & 839.079 & 839.368 & 839.316 & 855.961 & 856.455 & 858.249 \\
\hline & & 0.2 & 801.488 & 801.794 & 801.457 & 833.706 & 833.781 & 840.162 \\
\hline & 0.2 & 0.1 & 1498.803 & 1498.407 & 1498.297 & 1528.068 & 1526.689 & 1530.183 \\
\hline & & 0.2 & 1412.678 & 1411.727 & 1411.025 & 1469.096 & 1462.898 & 1474.891 \\
\hline \multirow[t]{4}{*}{0.1} & 0.1 & 0.1 & 853.032 & 853.147 & 853.095 & 869.733 & 870.057 & 871.706 \\
\hline & & 0.2 & 813.257 & 813.413 & 813.081 & 845.172 & 845.108 & 851.309 \\
\hline & 0.2 & 0.1 & 1506.389 & 1506.211 & 1506.101 & 1535.551 & 1534.422 & 1537.884 \\
\hline & & 0.2 & 1418.902 & 1418.331 & 1417.631 & 1475.145 & 1469.403 & 1481.404 \\
\hline \multirow[t]{4}{*}{0.2} & 0.1 & 0.1 & 870.670 & 870.705 & 870.655 & 887.107 & 887.350 & 888.783 \\
\hline & & 0.2 & 828.026 & 828.101 & 827.775 & 859.486 & 859.347 & 865.231 \\
\hline & 0.2 & 0.1 & 1514.343 & 1514.349 & 1514.240 & 1543.307 & 1542.389 & 1545.778 \\
\hline & & 0.2 & 1425.281 & 1424.974 & 1424.279 & 1481.171 & 1475.765 & 1487.688 \\
\hline
\end{tabular}

panel substrate is made of alumina $\left(\mathrm{Al}_{2} \mathrm{O}_{3}\right)$, and two piezoelectric layers are made of PZT-4. It should be noted that the results reported by Sayyaadi et al. ${ }^{[32,38]}$ were based on the HSDT. The excellent agreement between the results shows the accuracy of the current approach.

\subsubsection{Example 2}

Secondly, the free vibration of the laminated cross-ply and angle-ply PFG-CNTRC plates with simply supported boundary conditions is investigated. The fundamental frequencies are given in Table 5 for different CNT volume fractions, CNT distribution types, and electrical boundary conditions (EBC). Properties of the plate are set equal to $a=b=0.4 \mathrm{~m}, h=a / 20$, and $h_{\mathrm{p}}=h / 10$. The substrate is made of a multi-laminate of armchair SWCNT, and piezoelectric layers are PZT-5A. It can be seen that the present results agree well with those by Nguyen-Quang et al. ${ }^{[15]}$ based on the isogeometric approach and the HSDT. The maximum difference is only $1.41 \%$ for the case that $V_{\mathrm{CNT}}^{*}=0.28, \mathrm{FG}-\mathrm{X}$, and $\left[\mathrm{p} /-45^{\circ} / 45^{\circ} /-45^{\circ} /\right.$ $\left.-45^{\circ} / 45^{\circ} /-45^{\circ} / \mathrm{p}\right]$ configuration.

\subsection{Parametric studies}

\subsubsection{Free vibration of PFG-CNTRC doubly curved shell panels}

Parametric studies are carried out to understand the effects of material properties, geometric parameters, laminate configurations, and electrical boundaries on the free vibration responses of PFG-CNTRC doubly curved shell panels. The substrate of the panel is made of armchair SWCNT, and two piezoelectric layers are PZT-5A. Tables 6-8 list the fundamental frequencies of the CYL, spherical panel (SPH), and hyperbolic paraboloid (HPR) with various inlet parameters. It is observed from these tables that the distribution types of CNT have a significant effect on the stiffness of the panel. In detail, the FG-O panel has the lowest value of frequencies, while the FG-X panels have the highest ones. This conclusion is compatible with the conclusion of other researchers in the related studies in the literature. In three forms of doubly-curved shell panels, the HPR shell panels have the lowest frequencies, and the SPH shell panels have the highest frequencies. These results may become from the fact that SHP has a curvature effect, while the HPR has both positive and negative curvature that neutralize the effect of each other. These tables also reveal that the Opc of electrical boundary conditions always have higher frequencies than the Clc with all other parameters.

4.2.2 Effect of CNT distribution types

Figure 4 depicts the effect of distribution types of CNT on the fundamental frequencies of cross-ply PFG-CNTRC doubly curved shell panels for different $R_{x} / R_{y}$ ratio in case of the Opc condition. It is observed that the previous conclusions regarding the CNT distribution types are 
Table 5 Fundamental frequencies $\omega$ of square FG-CNTRC plates $\left(a=b, a / h=20, h_{\mathrm{p}}=h / 10\right.$, and $\left.R_{x}=R_{y}=\infty\right)$

\begin{tabular}{|c|c|c|c|c|c|c|c|c|}
\hline \multirow{3}{*}{$V_{\mathrm{CNT}}^{*}$} & \multirow{3}{*}{$\begin{array}{l}\text { FG } \\
\text { type }\end{array}$} & \multirow{3}{*}{$\mathrm{EBC}$} & \multicolumn{5}{|c|}{ Laminate configuration } & \\
\hline & & & \multicolumn{3}{|c|}{$\left[\mathrm{p} / 0^{\circ} / 90^{\circ} / 0^{\circ} / \mathrm{p}\right]$} & \multicolumn{3}{|c|}{$\left[\mathrm{p} /-45^{\circ} / 45^{\circ} /-45^{\circ} /-45^{\circ} / 45^{\circ} /-45^{\circ} / \mathrm{p}\right.$} \\
\hline & & & Present & Ref. [15] & Diff/\% & Present & Ref. [15] & Diff/\% \\
\hline \multirow{8}{*}{0.12} & \multirow[t]{2}{*}{ UD } & $\mathrm{Clc}$ & 587.093 & 583.510 & 0.61 & 662.572 & 656.538 & 0.92 \\
\hline & & Opc & 622.030 & 627.716 & -0.91 & 692.902 & 695.085 & -0.31 \\
\hline & \multirow[t]{2}{*}{ FG-X } & $\mathrm{Clc}$ & 593.270 & 588.372 & 0.83 & 667.328 & 658.696 & 1.31 \\
\hline & & Opc & 627.814 & 632.184 & -0.69 & 697.410 & 697.103 & 0.04 \\
\hline & \multirow[t]{2}{*}{ FG-V } & $\mathrm{Clc}$ & 585.306 & 581.714 & 0.62 & 661.675 & 655.606 & 0.93 \\
\hline & & Opc & 620.461 & 626.205 & -0.92 & 692.094 & 694.272 & -0.31 \\
\hline & \multirow[t]{2}{*}{ FG-O } & $\mathrm{Clc}$ & 580.947 & 578.737 & 0.38 & 657.878 & 654.510 & 0.51 \\
\hline & & Opc & 616.286 & 623.343 & -1.13 & 688.462 & 693.196 & -0.68 \\
\hline \multirow{8}{*}{0.17} & \multirow[t]{2}{*}{ UD } & $\mathrm{Clc}$ & 628.442 & 624.543 & 0.62 & 727.596 & 720.800 & 0.94 \\
\hline & & Opc & 660.887 & 665.615 & -0.71 & 754.830 & 755.388 & -0.07 \\
\hline & \multirow[t]{2}{*}{ FG-X } & $\mathrm{Clc}$ & 636.977 & 631.317 & 0.90 & 733.961 & 723.781 & 1.41 \\
\hline & & Opc & 668.952 & 671.913 & -0.44 & 760.925 & 758.217 & 0.36 \\
\hline & \multirow[t]{2}{*}{ FG-V } & Cloc & 625.826 & 621.914 & 0.63 & 726.448 & 719.594 & 0.95 \\
\hline & & Opc & 658.552 & 663.359 & -0.72 & 753.785 & 754.324 & -0.07 \\
\hline & \multirow[t]{2}{*}{ FG-O } & $\mathrm{Clc}$ & 620.132 & 618.126 & 0.32 & 721.527 & 718.247 & 0.46 \\
\hline & & Opc & 653.061 & 659.687 & -1.00 & 749.042 & 752.995 & -0.52 \\
\hline \multirow{8}{*}{0.28} & \multirow[t]{2}{*}{ UD } & $\mathrm{Clc}$ & 692.016 & 686.852 & 0.75 & 828.983 & 821.713 & 0.88 \\
\hline & & Opc & 720.749 & 723.150 & -0.33 & 851.843 & 850.524 & 0.16 \\
\hline & \multirow[t]{2}{*}{ FG-X } & $\mathrm{Clc}$ & 704.853 & 697.260 & 1.09 & 838.282 & 826.415 & 1.44 \\
\hline & & Opc & 733.029 & 732.991 & 0.01 & 860.872 & 855.093 & 0.68 \\
\hline & \multirow[t]{2}{*}{ FG-V } & $\mathrm{Clc}$ & 688.165 & 682.974 & 0.76 & 827.912 & 820.463 & 0.91 \\
\hline & & Opc & 717.276 & 719.788 & -0.35 & 850.901 & 849.465 & 0.17 \\
\hline & \multirow[t]{2}{*}{ FG-O } & $\mathrm{Clc}$ & 680.340 & 677.986 & 0.35 & 821.193 & 818.750 & 0.30 \\
\hline & & Opc & 709.665 & 714.904 & -0.73 & 844.364 & 847.767 & -0.40 \\
\hline
\end{tabular}

confirmed. The FG-X and FG-O have the highest and lowest frequencies, respectively. Figure 4 also shows that the value of the fundamental frequencies is minimum at the ratio $R_{x} / R_{y}=1$. This observation, maybe because of the curvature effect, is suppressed when the panels have the same value of positive and negative curvatures.

Moreover, the percentage change of frequency $\left(f_{\mathrm{PC}}\right)$ of the SPH panel is shown in Fig. 5 , where $f_{\mathrm{PC}}$ is defined as ${ }^{[39]}$

$$
f_{\mathrm{PC}}=\left(\frac{\omega_{\mathrm{FG}}-\omega_{\mathrm{UD}}}{\omega_{\mathrm{UD}}}\right) \times 100 \% .
$$

It can be seen from Fig. 5 that among four CNT distribution types, only FG-X shows the positive of $f_{\mathrm{PC}}$, while others show the negative of $f_{\mathrm{PC}}$. These results indicate that only the FG-X panel has higher stiffness than the UD panel, while UD is the simplest distribution type. 4.2.3 Effect of CNT volume fractions

Figure 6 indicates that the fundamental frequencies of the PFG-CNTRC doubly curved shell panels strongly increase with the increase of CNT volume fractions. The panels are set by $a / h=20, a / b=1, R_{x} / a=5, \mathrm{FG}-\mathrm{X}$ in Opc condition and $\left[\mathrm{p} / 0^{\circ} / 90^{\circ} / 0^{\circ} / \mathrm{p}\right]$ of configuration.

4.2.4 Effect of electrical boundary conditions

The natural frequency of laminated cross-ply FG-CNTRC doubly curved shell panels couped with closed and open piezoelectric layers are shown in Fig. 7. The parameters of the panels in this example are set by $a / h=20 ; a / b=1 ; R_{x} / a=5 ; \mathrm{FG}-\mathrm{X} ; V_{\mathrm{CNT}}^{*}=28 \%$. Figure 7 shows that the FG-CNTRC panels couped with the Opc always vibrate with the higher value of frequencies compared to the FG-CNTRC panels couped with the Clc because the Opc converts electric potential to mechanical energy while the Clc does not. 
Table 6 Fundamental frequencies $\omega$ of PFG-CNTRC-DCP $\left(a=b, R_{x} / a=5\right.$, and $\left.V_{\mathrm{CNT}}^{*}=0.12\right)$

\begin{tabular}{|c|c|c|c|c|c|c|c|c|}
\hline \multirow{3}{*}{ Shell panel } & \multirow{3}{*}{$\begin{array}{l}\text { FG } \\
\text { type }\end{array}$} & \multirow{3}{*}{$\mathrm{EBC}$} & \multicolumn{6}{|c|}{ Laminate configuration } \\
\hline & & & \multicolumn{2}{|c|}{$\left[\mathrm{p} / 0^{\circ} / \mathrm{p}\right]$} & \multicolumn{2}{|c|}{$\left[\mathrm{p} / 0^{\circ} / 90^{\circ} / 0^{\circ} / \mathrm{p}\right]$} & \multicolumn{2}{|c|}{$\left[\mathrm{p} /\left(-45^{\circ} / 45^{\circ} /-45^{\circ}\right)\right.$ as $\left./ \mathrm{p}\right]$} \\
\hline & & & $a / h=20$ & $a / h=100$ & $a / h=20$ & $a / h=100$ & $a / h=20$ & $a / h=100$ \\
\hline \multirow{10}{*}{$\begin{array}{c}\text { Cylindrical } \\
\text { (CYL, } \\
\left.R_{y}=\infty\right)\end{array}$} & \multirow[t]{2}{*}{ UD } & $\mathrm{Clc}$ & 592.304 & 154.618 & 593.607 & 159.006 & 687.170 & 235.130 \\
\hline & & Opc & 626.968 & 161.568 & 628.015 & 165.064 & 717.108 & 241.601 \\
\hline & \multirow[t]{2}{*}{ FG-A } & Clc & 567.175 & 147.982 & 589.313 & 156.416 & 687.365 & 235.617 \\
\hline & & Opc & 604.219 & 155.591 & 624.305 & 162.746 & 717.696 & 242.306 \\
\hline & \multirow[t]{2}{*}{ FG-V } & Clc & 571.471 & 153.409 & 594.398 & 161.032 & 685.268 & 234.439 \\
\hline & & Opc & 607.158 & 160.109 & 628.652 & 166.879 & 714.979 & 240.706 \\
\hline & \multirow[t]{2}{*}{ FG-X } & Clc & 630.839 & 161.458 & 599.683 & 160.097 & 692.114 & 236.894 \\
\hline & & Opc & 663.076 & 168.121 & 633.711 & 166.108 & 721.811 & 243.326 \\
\hline & \multirow[t]{2}{*}{ FG-O } & Clc & 550.429 & 147.529 & 587.575 & 157.968 & 682.292 & 233.388 \\
\hline & & Opc & 588.048 & 154.803 & 622.373 & 164.072 & 712.477 & 239.897 \\
\hline \multirow{10}{*}{$\begin{array}{l}\text { Spherical } \\
(\mathrm{SPH}, \\
\left.R_{y} / b=5\right)\end{array}$} & \multirow[t]{2}{*}{ UD } & $\mathrm{Clc}$ & 614.685 & 227.995 & 618.604 & 239.508 & 761.658 & 405.509 \\
\hline & & Opc & 648.704 & 234.979 & 651.724 & 244.267 & 791.004 & 413.788 \\
\hline & \multirow[t]{2}{*}{ FG-A } & Cloc & 586.781 & 221.745 & 611.821 & 236.324 & 762.835 & 406.178 \\
\hline & & Opc & 623.529 & 229.384 & 645.821 & 241.370 & 792.821 & 414.711 \\
\hline & \multirow[t]{2}{*}{ FG-V } & Clc & 598.356 & 229.060 & 622.037 & 242.409 & 758.969 & 404.781 \\
\hline & & Opc & 632.817 & 235.592 & 654.682 & 246.917 & 787.815 & 412.812 \\
\hline & \multirow[t]{2}{*}{ FG-X } & Clc & 651.893 & 232.777 & 624.478 & 240.418 & 767.182 & 408.539 \\
\hline & & Opc & 683.647 & 239.612 & 657.247 & 245.140 & 796.300 & 416.780 \\
\hline & \multirow[t]{2}{*}{ FG-O } & Clc & 574.487 & 223.264 & 612.812 & 238.726 & 756.211 & 402.522 \\
\hline & & Opc & 611.225 & 230.398 & 646.288 & 243.522 & 785.789 & 410.839 \\
\hline \multirow{10}{*}{$\begin{array}{l}\text { Hyperbolic } \\
\text { paraboloid } \\
(\mathrm{HPR}, \\
\left.R_{y} / b=-5\right)\end{array}$} & \multirow[t]{2}{*}{ UD } & Clc & 582.399 & 120.108 & 582.436 & 120.108 & 656.344 & 136.697 \\
\hline & & Opc & 617.059 & 127.493 & 617.099 & 127.493 & 686.410 & 143.221 \\
\hline & \multirow[t]{2}{*}{ FG-A } & $\mathrm{Clc}$ & 560.869 & 115.105 & 580.953 & 119.736 & 655.459 & 136.496 \\
\hline & & Opc & 597.416 & 122.836 & 615.839 & 127.165 & 685.614 & 143.038 \\
\hline & \multirow[t]{2}{*}{ FG-V } & Clc & 557.348 & 114.953 & 580.372 & 119.711 & 655.459 & 136.496 \\
\hline & & Opc & 593.608 & 122.670 & 615.246 & 127.140 & 685.614 & 143.038 \\
\hline & \multirow[t]{2}{*}{ FG-X } & Clc & 621.407 & 128.706 & 588.566 & 121.415 & 661.040 & 137.732 \\
\hline & & Opc & 653.585 & 135.620 & 622.840 & 128.725 & 690.861 & 144.209 \\
\hline & \multirow[t]{2}{*}{ FG-O } & Clc & 539.908 & 110.864 & 576.336 & 118.804 & 651.711 & 135.669 \\
\hline & & Opc & 577.610 & 118.829 & 611.398 & 126.266 & 682.029 & 142.240 \\
\hline
\end{tabular}

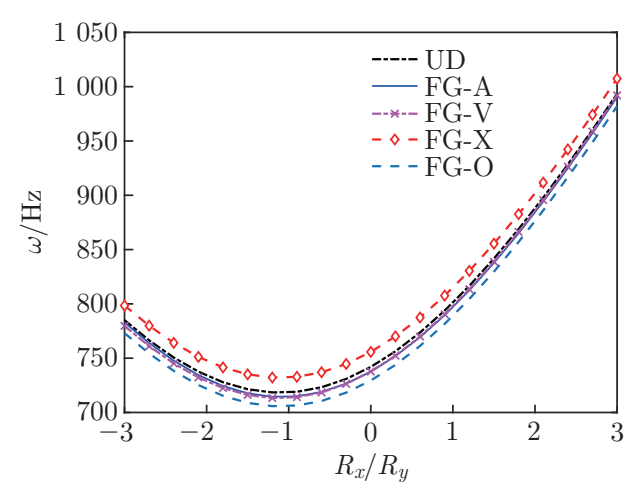

Fig. 4 Effects of distribution types of CNT on $\omega$ of PFG-CNTRC-DCP for different $R_{x} / R_{y}$ ratio $\left(\left[\mathrm{p} / 0^{\circ} / 90^{\circ} / 0^{\circ} / \mathrm{p}\right]\right.$, $a / h=20, a / b=1, R_{x} / a=5$, and $\left.V_{\mathrm{CNT}}^{*}=0.28\right)($ color online)

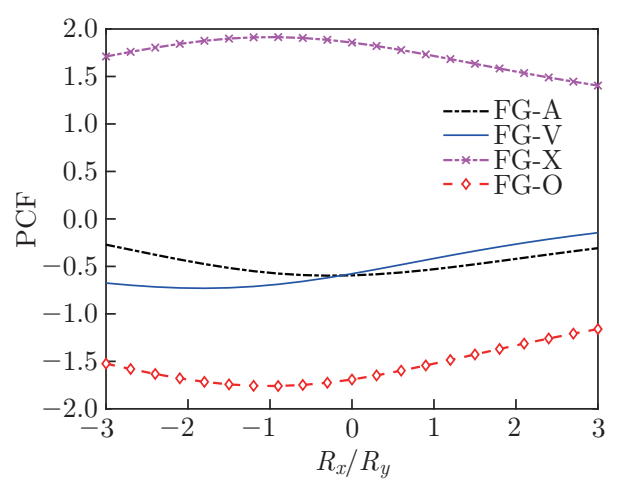

Fig. 5 Variation of PCF of PFG-CNTRC spherical shell panel with $R_{x} / R_{y}$ ratio (color online) 
Table 7 Fundamental frequencies $\omega$ of PFG-CNTRC-DCP $\left(a=b, R_{x} / a=5\right.$, and $\left.V_{\mathrm{CNT}}^{*}=0.17\right)$

\begin{tabular}{|c|c|c|c|c|c|c|c|c|}
\hline \multirow{3}{*}{ Shell panel } & \multirow{3}{*}{$\begin{array}{l}\text { FG } \\
\text { type }\end{array}$} & \multirow{3}{*}{$\mathrm{EBC}$} & \multicolumn{6}{|c|}{ Laminate configuration } \\
\hline & & & \multicolumn{2}{|c|}{$\left[\mathrm{p} / 0^{\circ} / \mathrm{p}\right]$} & \multicolumn{2}{|c|}{$\left[\mathrm{p} / 0^{\circ} / 90^{\circ} / 0^{\circ} / \mathrm{p}\right]$} & \multicolumn{2}{|c|}{$\left[\mathrm{p} /\left(-45^{\circ} / 45^{\circ} /-45^{\circ}\right)\right.$ as $\left./ \mathrm{p}\right]$} \\
\hline & & & $a / h=20$ & $a / h=100$ & $a / h=20$ & $a / h=100$ & $a / h=20$ & $a / h=100$ \\
\hline \multirow{10}{*}{$\begin{array}{c}\text { Cylindrical } \\
(\mathrm{CYL}, \\
\left.R_{y}=\infty\right)\end{array}$} & \multirow[t]{2}{*}{ UD } & $\mathrm{Clc}$ & 633.572 & 164.396 & 635.074 & 169.546 & 758.173 & 269.051 \\
\hline & & Opc & 665.777 & 170.885 & 667.020 & 175.122 & 784.980 & 274.812 \\
\hline & \multirow[t]{2}{*}{ FG-A } & Clc & 598.652 & 155.938 & 629.723 & 166.595 & 758.225 & 269.554 \\
\hline & & Opc & 633.715 & 163.155 & 662.292 & 172.434 & 785.445 & 275.530 \\
\hline & \multirow[t]{2}{*}{ FG-V } & Clc & 603.036 & 161.691 & 635.332 & 171.781 & 755.967 & 268.333 \\
\hline & & Opc & 636.713 & 167.967 & 667.202 & 177.165 & 782.551 & 273.892 \\
\hline & \multirow[t]{2}{*}{ FG-X } & $\mathrm{Clc}$ & 685.601 & 173.875 & 643.487 & 171.108 & 764.763 & 271.334 \\
\hline & & Opc & 714.948 & 180.017 & 674.977 & 176.629 & 791.309 & 277.054 \\
\hline & \multirow[t]{2}{*}{ FG-O } & Clc & 575.813 & 154.583 & 626.931 & 168.188 & 751.889 & 266.853 \\
\hline & & Opc & 611.696 & 161.471 & 659.343 & 173.815 & 778.967 & 272.653 \\
\hline \multirow{10}{*}{$\begin{array}{c}\text { Spherical } \\
(\mathrm{SPH}, \\
\left.R_{y} / b=5\right)\end{array}$} & \multirow[t]{2}{*}{ UD } & $\mathrm{Clc}$ & 656.589 & 240.614 & 661.155 & 254.209 & 849.365 & 469.563 \\
\hline & & Opc & 688.210 & 247.117 & 691.855 & 258.431 & 875.501 & 476.964 \\
\hline & \multirow[t]{2}{*}{ FG-A } & Clc & 619.120 & 233.156 & 653.114 & 250.730 & 850.514 & 470.304 \\
\hline & & Opc & 653.929 & 240.360 & 684.716 & 255.223 & 877.308 & 477.950 \\
\hline & \multirow[t]{2}{*}{ FG-V } & Clc & 631.141 & 240.924 & 664.426 & 257.588 & 846.377 & 468.865 \\
\hline & & Opc & 663.602 & 246.972 & 694.739 & 261.581 & 872.015 & 476.024 \\
\hline & \multirow[t]{2}{*}{ FG-X } & Clc & 706.953 & 247.435 & 669.331 & 255.599 & 856.660 & 473.443 \\
\hline & & Opc & 735.893 & 253.754 & 699.614 & 259.786 & 882.553 & 480.800 \\
\hline & \multirow[t]{2}{*}{ FG-O } & $\mathrm{Clc}$ & 601.151 & 234.135 & 653.377 & 253.302 & 842.404 & 465.845 \\
\hline & & Opc & 636.156 & 240.819 & 684.501 & 257.557 & 868.792 & 473.287 \\
\hline \multirow{10}{*}{$\begin{array}{l}\text { Hyperbolic } \\
\text { paraboloid } \\
(\mathrm{HPR} \\
\left.R_{y} / b=-5\right)\end{array}$} & \multirow[t]{2}{*}{ UD } & Clc & 623.421 & 128.847 & 623.462 & 128.848 & 720.586 & 150.857 \\
\hline & & Opc & 655.611 & 135.726 & 655.656 & 135.726 & 747.584 & 156.763 \\
\hline & \multirow[t]{2}{*}{ FG-A } & Clc & 592.150 & 121.654 & 621.209 & 128.289 & 719.455 & 150.584 \\
\hline & & $\mathrm{Opc}$ & 626.700 & 128.968 & 653.684 & 135.224 & 746.555 & 156.509 \\
\hline & \multirow[t]{2}{*}{ FG-V } & Clc & 588.329 & 121.488 & 620.521 & 128.260 & 719.454 & 150.584 \\
\hline & & Opc & 622.596 & 128.789 & 652.991 & 135.194 & 746.555 & 156.509 \\
\hline & \multirow[t]{2}{*}{ FG-X } & Clc & 676.025 & 140.546 & 631.932 & 130.654 & 726.872 & 152.244 \\
\hline & & Opc & 705.298 & 146.873 & 663.660 & 137.441 & 753.602 & 158.098 \\
\hline & \multirow[t]{2}{*}{ FG-O } & Clc & 564.824 & 116.046 & 615.213 & 127.073 & 714.596 & 149.505 \\
\hline & & Opc & 600.798 & 123.644 & 647.887 & 134.043 & 741.873 & 155.462 \\
\hline
\end{tabular}

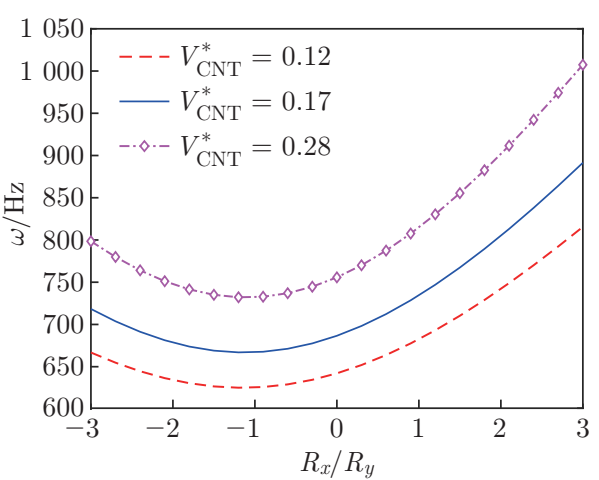

Fig. 6 Effect of $V_{\mathrm{CNT}}^{*}$ on fundamental frequencies $\omega$ of PFG-CNTRC shell panel (color online)

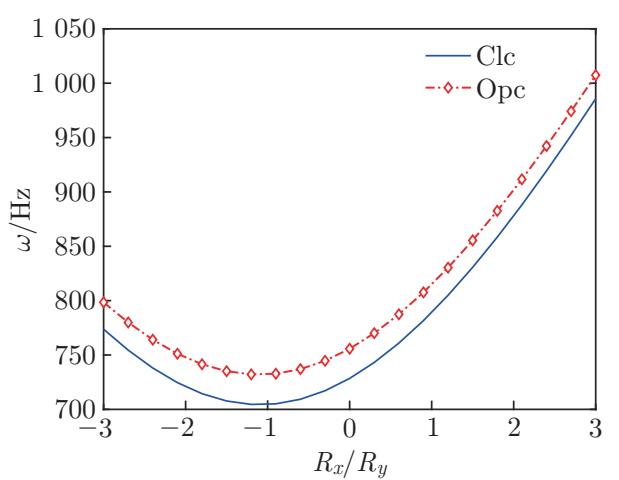

Fig. 7 Effect of electrical boundary conditions on $\omega$ of PFG-CNTRC-DCP (color online)

\subsubsection{Effect of curvature of shell panels}

The effect of radii of curvature on the natural frequencies of the SPH, CYL, and HPR shell panels are shown in Figs. 8 to 10, respectively. It can be seen from these figures that with the 
Table 8 Fundamental frequencies $\omega$ of PFG-CNTRC-DCP $\left(a=b, R_{x} / a=5\right.$, and $\left.V_{\mathrm{CNT}}^{*}=0.28\right)$

\begin{tabular}{|c|c|c|c|c|c|c|c|c|}
\hline \multirow{3}{*}{ Shell panel } & \multirow{3}{*}{$\begin{array}{l}\text { FG } \\
\text { type }\end{array}$} & \multirow{3}{*}{$\mathrm{EBC}$} & \multicolumn{6}{|c|}{ Laminate configuration } \\
\hline & & & \multicolumn{2}{|c|}{$\left[\mathrm{p} / 0^{\circ} / \mathrm{p}\right]$} & \multicolumn{2}{|c|}{$\left[\mathrm{p} / 0^{\circ} / 90^{\circ} / 0^{\circ} / \mathrm{p}\right]$} & \multicolumn{2}{|c|}{$\left[\mathrm{p} /\left(-45^{\circ} / 45^{\circ} /-45^{\circ}\right)\right.$ as $\left./ \mathrm{p}\right]$} \\
\hline & & & $a / h=20$ & $a / h=100$ & $a / h=20$ & $a / h=100$ & $a / h=20$ & $a / h=100$ \\
\hline \multirow{10}{*}{$\begin{array}{l}\text { Cylindrical } \\
\quad(\mathrm{CYL}, \\
\left.R_{y}=\infty\right)\end{array}$} & \multirow[t]{2}{*}{ UD } & $\mathrm{Clc}$ & 696.079 & 176.288 & 697.771 & 182.254 & 869.286 & 323.219 \\
\hline & & Opc & 724.661 & 182.264 & 726.093 & 187.293 & 891.715 & 328.106 \\
\hline & \multirow[t]{2}{*}{ FG-A } & $\mathrm{Clc}$ & 645.376 & 165.016 & 691.385 & 179.245 & 869.397 & 323.703 \\
\hline & & Opc & 677.457 & 171.831 & 720.359 & 184.516 & 892.263 & 328.795 \\
\hline & \multirow[t]{2}{*}{ FG-V } & $\mathrm{Clc}$ & 648.885 & 170.533 & 696.794 & 184.631 & 867.253 & 322.606 \\
\hline & & Opc & 679.588 & 176.355 & 725.199 & 189.519 & 889.487 & 327.300 \\
\hline & \multirow[t]{2}{*}{ FG-X } & $\mathrm{Clc}$ & 770.002 & 190.798 & 710.510 & 184.890 & 878.859 & 326.396 \\
\hline & & Opc & 795.215 & 196.326 & 738.290 & 189.858 & 901.030 & 331.240 \\
\hline & \multirow[t]{2}{*}{ FG-O } & $\mathrm{Clc}$ & 611.394 & 161.438 & 686.367 & 180.466 & 861.225 & 320.333 \\
\hline & & Opc & 644.696 & 167.950 & 715.260 & 185.559 & 883.954 & 325.259 \\
\hline \multirow{10}{*}{$\begin{array}{c}\text { Spherical } \\
\quad(\mathrm{SPH} \\
\left.R_{y} / b=5\right)\end{array}$} & \multirow[t]{2}{*}{ UD } & $\mathrm{Clc}$ & 717.463 & 250.215 & 722.558 & 266.395 & 987.409 & 570.737 \\
\hline & & Opc & 745.644 & 256.334 & 749.826 & 270.100 & 1009.173 & 577.071 \\
\hline & \multirow[t]{2}{*}{ FG-A } & $\mathrm{Clc}$ & 665.080 & 241.344 & 713.761 & 263.385 & 988.655 & 571.585 \\
\hline & & Opc & 697.073 & 248.260 & 741.898 & 267.320 & 1011.083 & 578.149 \\
\hline & \multirow[t]{2}{*}{ FG-V } & $\mathrm{Clc}$ & 675.734 & 248.955 & 724.873 & 270.667 & 984.732 & 570.292 \\
\hline & & Opc & 705.374 & 254.601 & 751.949 & 274.180 & 1006.032 & 576.397 \\
\hline & \multirow[t]{2}{*}{ FG-X } & $\mathrm{Clc}$ & 789.565 & 261.511 & 735.169 & 269.205 & 997.876 & 576.086 \\
\hline & & Opc & 814.537 & 267.361 & 761.933 & 272.869 & 1019.404 & 582.372 \\
\hline & \multirow[t]{2}{*}{ FG-O } & $\mathrm{Clc}$ & 635.961 & 240.559 & 711.788 & 265.641 & 978.484 & 565.926 \\
\hline & & Opc & 668.532 & 246.925 & 739.573 & 269.371 & 1000.521 & 572.303 \\
\hline \multirow{10}{*}{$\begin{array}{l}\text { Hyperbolic } \\
\text { paraboloid } \\
\quad(\mathrm{HPR} \\
\left.R_{y} / b=-5\right)\end{array}$} & \multirow[t]{2}{*}{ UD } & $\mathrm{Clc}$ & 686.512 & 142.888 & 686.568 & 142.888 & 820.668 & 174.254 \\
\hline & & Opc & 715.023 & 149.054 & 715.082 & 149.054 & 843.322 & 179.332 \\
\hline & \multirow[t]{2}{*}{ FG-A } & $\mathrm{Clc}$ & 638.958 & 131.868 & 683.163 & 141.999 & 819.621 & 173.881 \\
\hline & & Opc & 670.478 & 138.592 & 712.050 & 148.236 & 842.404 & 178.979 \\
\hline & \multirow[t]{2}{*}{ FG-V } & $\mathrm{Clc}$ & 634.839 & 131.687 & 682.322 & 141.962 & 819.621 & 173.881 \\
\hline & & $\mathrm{Opc}$ & 666.077 & 138.397 & 711.212 & 148.199 & 842.404 & 178.979 \\
\hline & \multirow[t]{2}{*}{ FG-X } & $\mathrm{Clc}$ & 761.021 & 159.902 & 699.305 & 145.597 & 829.855 & 176.247 \\
\hline & & Opc & 786.116 & 165.428 & 727.267 & 151.652 & 852.241 & 181.268 \\
\hline & \multirow[t]{2}{*}{ FG-O } & $\mathrm{Clc}$ & 600.719 & 123.820 & 674.977 & 140.327 & 812.987 & 172.392 \\
\hline & & $\mathrm{Opc}$ & 634.061 & 130.896 & 704.078 & 146.602 & 835.950 & 177.524 \\
\hline
\end{tabular}

increase of $R_{x} / a$ ratio, the frequencies of SPH and CYL decrease while the frequencies of HPR increase. The frequencies of all three types of shell panels are approximately equal to those of the plate with the corresponding input parameters when the value of $R_{x} / a$ ratio reaches 20 . This observation once again confirms that the opposite curvature will reduce the stiffness of the shells.

4.2.6 Effect of piezoelectric layer thickness

In order to investigate the effect of piezoelectric layer thickness on the free vibration response of the composite shell panel, the variation of percentage difference in natural frequency $\beta$ is defined as ${ }^{[40]}$

$$
\beta=\frac{\omega_{\text {with piezoelectric layer }}-\omega_{\text {without piezoelectric layer }}}{\omega_{\text {without piezoelectric layer }}} \times 100 \% .
$$

Figures 11, 12, and 13 present the percentage difference in natural frequency $\beta$ versus the $h_{\mathrm{p}} / h$ ratios for different forms of the panel, distribution types of CNT, and CNT volume fractions. It is observed from these figures that the HPR panel has a higher value of $\beta$ than CYL and SPH panel, the panel reinforced with lower CNT volume fractions has a higher value of $\beta$, among five CNT distribution types, the FG-O panel has the highest value of $\beta$ while the FG-X has lowest one. It can be concluded that the piezoelectric effects are more effective in the case of smaller stiffness panels rather than the case of greater stiffness ones. Furthermore, 


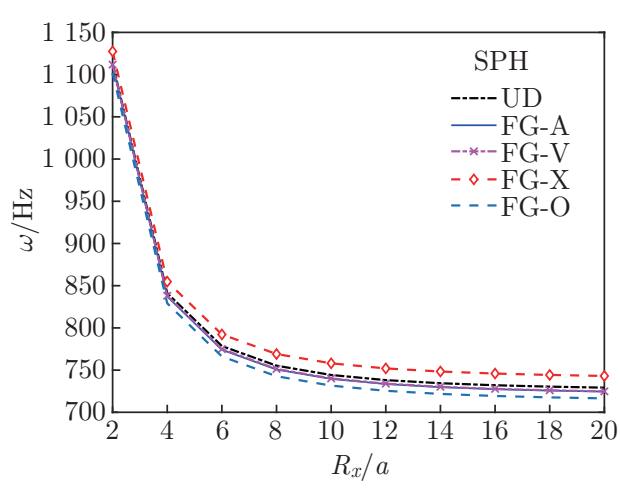

Fig. 8 Effect of radius of curvature on $\omega$ of PFG-CNTRC spherical shell panels $\left(a / h=20, a / b=1, V_{\mathrm{CNT}}^{*}=0.28\right.$, $R_{y}=R_{x},\left[\mathrm{p} / 0^{\circ} / 90^{\circ} / 0^{\circ} / \mathrm{p}\right]$ ) (color online)

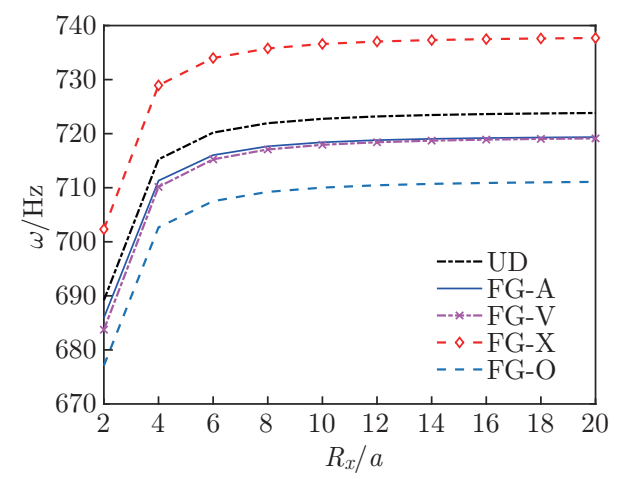

Fig. 10 Effect of radius of curvature on $\omega$ of PFG-CNTRC HPR shell panels $\left(a / h=20, a / b=1, V_{\mathrm{CNT}}^{*}=0.28\right.$, $R_{y}=-R_{x},\left[\mathrm{p} / 0^{\circ} / 90^{\circ} / 0^{\circ} / \mathrm{p}\right]$ ) (color online)

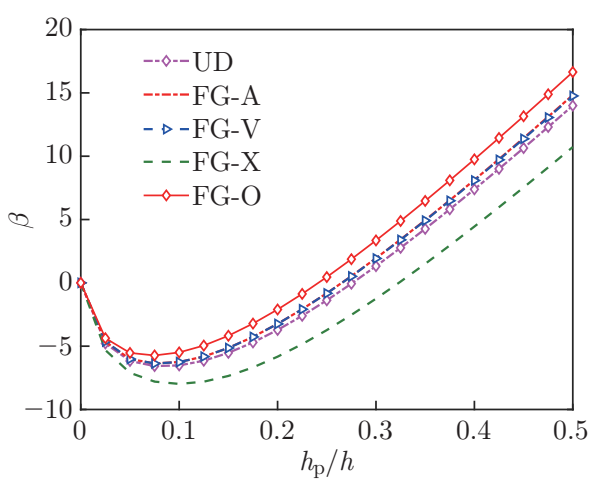

Fig. 12 Variation of $\beta$ of spherical panel versus $h_{\mathrm{p}} / h$ ratios for different CNT distribution types $(a / h=20, a / b=1$, $R_{x} / a=R_{y} / b=5, V_{\mathrm{CNT}}^{*}=0.28$, $\left.\left[\mathrm{p} / 0^{\circ} / 90^{\circ} / 0^{\circ} / \mathrm{p}\right]\right)$ (color online)

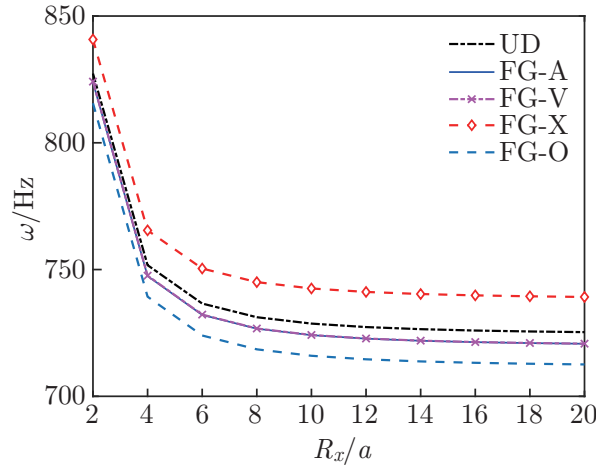

Fig. 9 Effect of radius of curvature on $\omega$ of PFG-CNTRC cylindrical shell panels $\left(a / h=20, a / b=1, V_{\mathrm{CNT}}^{*}=0.28\right.$, $R_{y}=\infty,\left[\mathrm{p} / 0^{\circ} / 90^{\circ} / 0^{\circ} / \mathrm{p}\right]$ ) (color online)

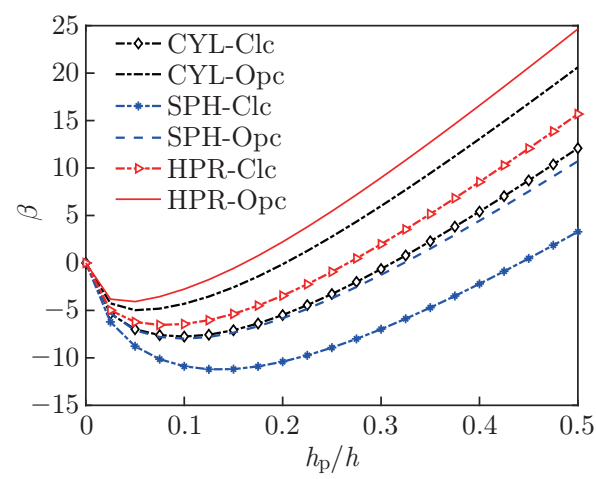

Fig. 11 Variation of $\beta$ of the PFG-CNTRCDCP versus $h_{\mathrm{p}} / h$ ratios for electrical boundary conditions $(a / h=20$, $a / b=1, R_{x} / a=5, \mathrm{FG}-\mathrm{X}, V_{\mathrm{CNT}}^{*}=$ $\left.0.28,\left[0^{\circ} / 90^{\circ} / 0^{\circ}\right]\right)$ (color online)

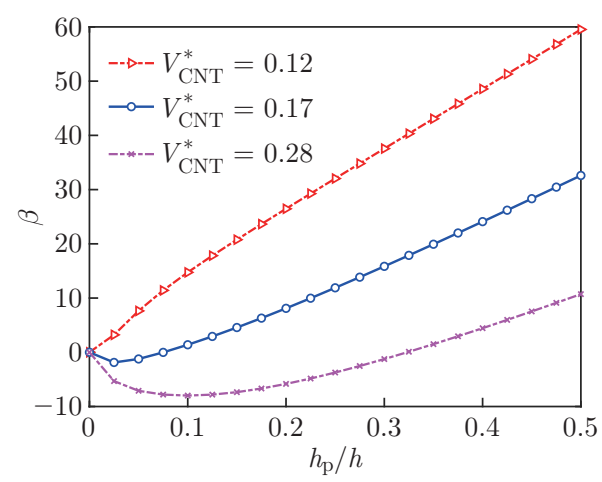

Fig. 13 Variation of $\beta$ of spherical panel versus $h_{\mathrm{p}} / h$ ratios for different CNT volume fractions $(a / h=20, a / b=$ $1, R_{x} / a=R_{y} / b=5, \quad$ FG-X, $\left.\left[\mathrm{p} / 0^{\circ} / 90^{\circ} / 0^{\circ} / \mathrm{p}\right]\right)$ (color online) 
these figures also indicate that increasing the thickness of piezoelectric layers from zero to a specific value leads to a decrement of $\beta$. It can be explained by the fact that the piezoelectric material has a higher mass density and lower elastic moduli compared with the core material. After that specific value of $h_{\mathrm{p}} / h$, the percentage difference in natural frequency $\beta$ increases by the increment of piezoelectric layers thickness because the electromechanical coupling effect increases with an increase in $h_{\mathrm{p}} / h$ and should increase the value of $\beta$. The electromechanical coupling effect is lower than the combined effects of the increase in the mass density, and the decrease in the stiffness results in negative values of $\beta$ for values of $h_{\mathrm{p}} / h$.

\section{Conclusions}

In this paper, an analytical solution based on the four-variable shear deformation refined theory is developed for carrying out the free vibration of the laminated functionally graded nanotube-reinforced composite doubly curved shell panels with surface-bonded piezoelectric layers. Comparison studies validate the accuracy of the model. Numerical results are provided to explore the effects of the CNT volume fraction, the CNT distribution type, the thickness of the piezoelectric layers, laminate configurations, and mechanical and electrical boundary conditions on the natural frequencies of the hybrid panels. Through the present formulation and numerical results, some conclusions can be drawn. (i) The volume fraction of CNT has substantial effects on dynamic responses of PFG-CNTRC panels. (ii) The natural frequencies of PFG-CNTRC panel with Opc electrical boundary condition are always higher than those with the Clc case when all other inlet parameters are the same. (iii) CNT reinforcements distributed close to top and bottom are more efficient than those distributed near the midplane for increasing the stiffness of the panels.

Open Access This article is licensed under a Creative Commons Attribution 4.0 International License, which permits use, sharing, adaptation, distribution and reproduction in any medium or format, as long as you give appropriate credit to the original author(s) and the source, provide a link to the Creative Commons licence, and indicate if changes were made. To view a copy of this licence, visit http://creativecommons.org/licenses/by/4.0/.

\section{References}

[1] ALIBEIGLOO, A. Static analysis of functionally graded carbon nanotube-reinforced composite plate embedded in piezoelectric layers by using theory of elasticity. Composite Structures, 95, 612-622 (2013)

[2] ALIBEIGLOO, A. Elasticity solution of functionally graded carbon-nanotube-reinforced composite cylindrical panel with piezoelectric sensor and actuator layers. Smart Materials and Structures, 22(7), 075013 (2013)

[3] ALIBEIGLOO, A. Three-dimensional thermoelasticity solution of functionally graded carbon nanotube reinforced composite plate embedded in piezoelectric sensor and actuator layers. Composite Structures, 118, 482-495 (2014)

[4] ALIBEIGLOO, A. Thermoelastic analysis of functionally graded carbon nanotube reinforced composite cylindrical panel embedded in piezoelectric sensor and actuator layers. Composites Part B: Engineering, 98, 225-243 (2016)

[5] ALIBEIGLOO, A. Free vibration analysis of functionally graded carbon nanotube-reinforced composite cylindrical panel embedded in piezoelectric layers by using theory of elasticity. European Journal of Mechanics-A/Solids, 44, 104-115 (2014)

[6] WU, C. P. and CHANG, S. K. Stability of carbon nanotube-reinforced composite plates with surface-bonded piezoelectric layers and under bi-axial compression. Composite Structures, 111, 587-601 (2014) 
[7] NASihAtgozar, M., DAGHigh, V., ESKAndARI, M., NIKBIN, K., and SimoneAU, A. Buckling analysis of piezoelectric cylindrical composite panels reinforced with carbon nanotubes. International Journal of Mechanical Sciences, 107, 69-79 (2016)

[8] ANSARI, R., POURASHRAF, T., GHOLAMI, R., and SHAHABODINI, A. Analytical solution for nonlinear postbuckling of functionally graded carbon nanotube-reinforced composite shells with piezoelectric layers. Composites Part B: Engineering, 90, 267-277 (2016)

[9] RAFIEE, M., HE, X., and LIEW, K. Non-linear dynamic stability of piezoelectric functionally graded carbon nanotube-reinforced composite plates with initial geometric imperfection. International Journal of Non-Linear Mechanics, 59, 37-51 (2014)

[10] RAFIEE, M., LIU, X., HE, X., and KITIPORNCHAI, S. Geometrically nonlinear free vibration of shear deformable piezoelectric carbon nanotube/fiber/polymer multiscale laminated composite plates. Journal of Sound and Vibration, 333(14), 3236-3251 (2014)

[11] KIANI, Y. Free vibration of functionally graded carbon nanotube reinforced composite plates integrated with piezoelectric layers. Computers \& Mathematics with Applications, 72(9), 24332449 (2016)

[12] SHARMA, A., KUMAR, A., SUSHEEL, C., and KUMAR, R. Smart damping of functionally graded nanotube reinforced composite rectangular plates. Composite Structures, 155, 29-44 (2016)

[13] KOLAHCHI, R., ZAREI, M. S., HAJMOHAMMAD, M. H., NOURI, A., HAJMOHAMMAD, M. H., and NOURI, A. Wave propagation of embedded viscoelastic FG-CNT-reinforced sandwich plates integrated with sensor and actuator based on refined zigzag theory. International Journal of Mechanical Sciences, 130, 534-545 (2017)

[14] SETOODEH, A., SHOJAEE, M., and MALEKZADEH, P. Application of transformed differential quadrature to free vibration analysis of FG-CNTRC quadrilateral spherical panel with piezoelectric layers. Computer Methods in Applied Mechanics and Engineering, 335, 510-537 (2018)

[15] NGUYEN-QUANG, K., VO-DUY, T., DANG-TRUNG, H., and NGUYEN-THOI, T. An isogeometric approach for dynamic response of laminated FG-CNT reinforced composite plates integrated with piezoelectric layers. Computer Methods in Applied Mechanics and Engineering, 332, 25-46 (2018)

[16] SELIM, B., YIN, B., and LIEW, K. Impact analysis of CNT-reinforced composite plates integrated with piezoelectric layers based on Reddy's higher-order shear deformation theory. Composites Part B: Engineering, 136, 10-19 (2018)

[17] SONG, Z., ZHANG, L., and LIEW, K. Active vibration control of CNT reinforced functionally graded plates based on a higher-order shear deformation theory. International Journal of Mechanical Sciences, 105, 90-101 (2016)

[18] SELIM, B., ZHANG, L., and LIEW, K. Active vibration control of CNT-reinforced composite plates with piezoelectric layers based on Reddy's higher-order shear deformation theory. Composite Structures, 163, 350-364 (2017)

[19] ZHANG, L., SONG, Z., and LIEW, K. Optimal shape control of CNT reinforced functionally graded composite plates using piezoelectric patches. Composites Part B: Engineering, 85, 140149 (2016)

[20] TRAn, M. T., NGUYEn, V. L., PHAM, S. D., and RUNGAMORNRAT, J. Free vibration of stiffened functionally graded circular cylindrical shell resting on Winkler-Pasternak foundation with different boundary conditions under thermal environment. Acta Mechanica, 231(6), 25452564 (2020)

[21] BAGHLANI, A., KHAYAT, M., and DEHGHAN, S. M. Free vibration analysis of FGM cylindrical shells surrounded by Pasternak elastic foundation in thermal environment considering fluidstructure interaction. Applied Mathematical Modelling, 78, 550-575 (2020)

[22] SONG, Z., ZHANG, L., and LIEW, K. Active vibration control of CNT-reinforced composite cylindrical shells via piezoelectric patches. Composite Structures, 158, 92-100 (2016)

[23] ZHU, C. S., FANG, X. Q., LIU, J. X., and LI, H. Y. Surface energy effect on nonlinear free vibration behavior of orthotropic piezoelectric cylindrical nano-shells. European Journal of MechanicsA/Solids, 66, 423-432 (2017) 
[24] ZHU, C., FANG, X., and LIU, J. A new approach for smart control of size-dependent nonlinear free vibration of viscoelastic orthotropic piezoelectric doubly-curved nanoshells. Applied Mathematical Modelling, 77, 137-168 (2020)

[25] TRAN, H. Q., VAN, T., TRAN, M. T., and NGUYEN-TRI, P. A new four-variable refined plate theory for static analysis of smart laminated functionally graded carbon nanotube reinforced composite plates. Mechanics of Materials, 142, 103294 (2020)

[26] HUU, Q. T., MINH, T. T., and VAN, T. V. Free vibration analysis of smart laminated functionally graded CNT reinforced composite plates via new four-variable refined plate theory. Materials, $\mathbf{1 2}(22), 3675(2019)$

[27] ARANI, A. G., KIANI, F., and AFSHARI, H. Free and forced vibration analysis of laminated functionally graded CNT-reinforced composite cylindrical panels. Journal of Sandwich Structures \& Materials, 1099636219830787 (2019)

[28] LEI, Z., ZHANG, L., and LIEW, K. Analysis of laminated CNT reinforced functionally graded plates using the element-free $k p$-Ritz method. Composites Part B: Engineering, 84, 211-221 (2016)

[29] VAN, T. V., HUU, Q. T., and MINH, T. T. Free vibration analysis of laminated functionally graded carbon nanotube-reinforced composite doubly curved shallow shell panels using a new four-variable refined theory. Journal of Composites Science, 3(4), 104 (2019)

[30] QATU, M. S. Vibration of Laminated Shells and Plates, Elsevier, Oxford (2004)

[31] SHIYEKAR, S. and KANT, T. Higher order shear deformation effects on analysis of laminates with piezoelectric fibre reinforced composite actuators. Composite Structures, 93(12), 3252-3261 (2011)

[32] SAYYAADI, H., RAHNAMA, F., and FARSANGI, M. A. A. Energy harvesting via shallow cylindrical and spherical piezoelectric panels using higher order shear deformation theory. Composite Structures, 147, 155-167 (2016)

[33] ZENKOUR, A. M. and ALGHANMI, R. A. Bending of exponentially graded plates integrated with piezoelectric fiber-reinforced composite actuators resting on elastic foundations. European Journal of Mechanics-A/Solids, 75, 461-471 (2019)

[34] WANG, Q., SHAO, D., and QIN, B. A simple first-order shear deformation shell theory for vibration analysis of composite laminated open cylindrical shells with general boundary conditions. Composite Structures, 184, 211-232 (2018)

[35] REDDY, J. N. Mechanics of Laminated Composite Plates and Shells: Theory and Analysis, CRC Press, Boca Raton (2004)

[36] FARSANGI, M. A., SAIDI, A., and BATRA, R. Analytical solution for free vibrations of moderately thick hybrid piezoelectric laminated plates. Journal of Sound and Vibration, 332(22), 5981-5998 (2013)

[37] ROUZEGAR, J. and ABAD, F. Free vibration analysis of FG plate with piezoelectric layers using four-variable refined plate theory. Thin-Walled Structures, 89, 76-83 (2015)

[38] SAYYAADI, H. and FARSANGI, M. A. A. An analytical solution for dynamic behavior of thick doubly curved functionally graded smart panels. Composite Structures, 107, 88-102 (2014)

[39] POURESMAEELI, S. and FAZELZADEH, S. Frequency analysis of doubly curved functionally graded carbon nanotube-reinforced composite panels. Acta Mechanica, 227(10), 2765-2794 (2016)

[40] FARSANGI, M. A. and SAIDI, A. Levy type solution for free vibration analysis of functionally graded rectangular plates with piezoelectric layers. Smart Materials and Structures, 21(9), 094017 (2012)

\section{Appendix A}

$$
\begin{aligned}
\left(\begin{array}{c}
N_{x x} \\
N_{x y}
\end{array}\right)= & \sum_{k=1}^{n} \int_{z_{k}}^{z_{k+1}}\left(1+\frac{z}{R_{y}}\right)\left(\begin{array}{c}
\sigma_{x x}^{k} \\
\tau_{x y}^{k}
\end{array}\right) \mathrm{d} z-\int_{-h / 2-h_{\mathrm{p}}}^{-h / 2}\left(1+\frac{z}{R_{y}}\right)\left(\begin{array}{c}
\sigma_{x x}^{\text {pie }} \\
\tau_{x y}^{\text {pie }}
\end{array}\right)^{\mathrm{B}} \mathrm{d} z \\
& -\int_{h / 2}^{h / 2+h_{\mathrm{p}}}\left(1+\frac{z}{R_{y}}\right)\left(\begin{array}{c}
\sigma_{x x}^{\text {pie }} \\
\tau_{x y}^{\text {pie }}
\end{array}\right)^{\mathrm{T}} \mathrm{d} z
\end{aligned}
$$




$$
\begin{aligned}
& \left(\begin{array}{c}
N_{y y} \\
N_{y x}
\end{array}\right)=\sum_{k=1}^{n} \int_{z_{k}}^{z_{k+1}}\left(1+\frac{z}{R_{x}}\right)\left(\begin{array}{c}
\sigma_{y y}^{k} \\
\tau_{y x}^{k}
\end{array}\right) \mathrm{d} z-\int_{-h / 2-h_{\mathrm{p}}}^{-h / 2}\left(1+\frac{z}{R_{x}}\right)\left(\begin{array}{c}
\sigma_{y y}^{\text {pie }} \\
\tau_{y x}^{\text {pie }}
\end{array}\right)^{\mathrm{B}} \mathrm{d} z \\
& -\int_{h / 2}^{h / 2+h_{\mathrm{p}}}\left(1+\frac{z}{R_{x}}\right)\left(\begin{array}{c}
\sigma_{y y}^{\text {pie }} \\
\tau_{y x}^{\text {pie }}
\end{array}\right)^{\mathrm{T}} \mathrm{d} z \\
& \left(\begin{array}{c}
M_{x x}^{\mathrm{b}} \\
M_{x y}^{\mathrm{b}}
\end{array}\right)=\sum_{k=1}^{n} \int_{z_{k}}^{z_{k+1}}\left(1+\frac{z}{R_{y}}\right)\left(\begin{array}{c}
\sigma_{x x}^{k} \\
\sigma_{x y}^{k}
\end{array}\right) z \mathrm{~d} z-\int_{-h / 2-h_{\mathrm{p}}}^{-h / 2}\left(1+\frac{z}{R_{y}}\right)\left(\begin{array}{c}
\sigma_{x x}^{\text {pie }} \\
\tau_{x y}^{\text {pie }}
\end{array}\right)^{\mathrm{B}} z \mathrm{~d} z \\
& -\int_{h / 2}^{h / 2+h_{\mathrm{p}}}\left(1+\frac{z}{R_{y}}\right)\left(\begin{array}{c}
\sigma_{x x}^{\text {pie }} \\
\tau_{x y}^{\text {pie }}
\end{array}\right)^{\mathrm{T}} z \mathrm{~d} z, \\
& \left(\begin{array}{c}
M_{y y}^{\mathrm{b}} \\
M_{y x}^{\mathrm{b}}
\end{array}\right)=\sum_{k=1}^{n} \int_{z_{k}}^{z_{k+1}}\left(1+\frac{z}{R_{x}}\right)\left(\begin{array}{c}
\sigma_{y y}^{k} \\
\sigma_{y x}^{k}
\end{array}\right) z \mathrm{~d} z-\int_{-h / 2-h_{\mathrm{p}}}^{-h / 2}\left(1+\frac{z}{R_{x}}\right)\left(\begin{array}{c}
\sigma_{y y}^{\text {pie }} \\
\tau_{y x}^{\text {pie }}
\end{array}\right)^{\mathrm{B}} z \mathrm{~d} z \\
& -\int_{h / 2}^{h / 2+h_{\mathrm{p}}}\left(1+\frac{z}{R_{x}}\right)\left(\begin{array}{c}
\sigma_{y y}^{\text {pie }} \\
\tau_{y x}^{\text {pie }}
\end{array}\right)^{\mathrm{T}} z \mathrm{~d} z, \\
& \left(\begin{array}{l}
M_{x x}^{\mathrm{s}} \\
M_{x y}^{\mathrm{s}}
\end{array}\right)=\sum_{k=1}^{n} \int_{z_{k}}^{z_{k+1}}\left(1+\frac{z}{R_{y}}\right)\left(\begin{array}{c}
\sigma_{x x}^{k} \\
\sigma_{x y}^{k}
\end{array}\right) f(z) \mathrm{d} z-\int_{-h / 2-h_{\mathrm{p}}}^{h / 2}\left(1+\frac{z}{R_{y}}\right)\left(\begin{array}{l}
\sigma_{x x}^{\mathrm{pix}} \\
\tau_{x y}^{\text {pie }}
\end{array}\right)^{\mathrm{B}} f(z) \mathrm{d} z \\
& -\int_{h / 2}^{h / 2+h_{\mathrm{p}}}\left(1+\frac{z}{R_{y}}\right)\left(\begin{array}{c}
\sigma_{x x}^{\text {pie }} \\
\tau_{x y}^{\text {pie }}
\end{array}\right)^{\mathrm{T}} f(z) \mathrm{d} z \\
& \left(\begin{array}{l}
M_{y y}^{\mathrm{s}} \\
M_{y x}^{\mathrm{s}}
\end{array}\right)=\sum_{k=1}^{n} \int_{z_{k}}^{z_{k+1}}\left(1+\frac{z}{R_{x}}\right)\left(\begin{array}{c}
\sigma_{y y}^{k} \\
\sigma_{y x}^{k}
\end{array}\right) f(z) \mathrm{d} z-\int_{-h / 2-h_{\mathrm{p}}}^{-h / 2}\left(1+\frac{z}{R_{x}}\right)\left(\begin{array}{l}
\sigma_{y y}^{\text {pie }} \\
\tau_{y x}^{\text {pie }}
\end{array}\right)^{\mathrm{B}} f(z) \mathrm{d} z \\
& -\int_{h / 2}^{h / 2+h_{\mathrm{p}}}\left(1+\frac{z}{R_{x}}\right)\left(\begin{array}{c}
\sigma_{y y}^{\text {pie }} \\
\tau_{y x}^{\text {pie }}
\end{array}\right)^{\mathrm{T}} f(z) \mathrm{d} z, \\
& Q_{x \mathrm{~s}}=\sum_{k=1}^{n} \int_{z_{k}}^{z_{k+1}}\left(1+\frac{z}{R_{y}}\right) \tau_{x z}^{k} \mathrm{~d} z-\int_{-h / 2-h_{\mathrm{p}}}^{-h / 2}\left(1+\frac{z}{R_{y}}\right) \tau_{x z}^{\mathrm{pieB}} \mathrm{d} z-\int_{h / 2}^{h / 2+h_{\mathrm{p}}}\left(1+\frac{z}{R_{y}}\right) \tau_{x z}^{\mathrm{pie \textrm {T }}} \mathrm{d} z, \\
& Q_{y \mathrm{~s}}=\sum_{k=1}^{n} \int_{z_{k}}^{z_{k+1}}\left(1+\frac{z}{R_{x}}\right) \tau_{y z}^{k} \mathrm{~d} z-\int_{-h / 2-h_{\mathrm{p}}}^{-h / 2}\left(1+\frac{z}{R_{y}}\right) \tau_{y z}^{\mathrm{pieB}} \mathrm{d} z-\int_{h / 2}^{h / 2+h_{\mathrm{p}}}\left(1+\frac{z}{R_{y}}\right) \tau_{y z}^{\mathrm{pieT}} \mathrm{d} z .
\end{aligned}
$$

\section{Appendix B1}

$$
\begin{aligned}
m_{11}= & -\left(\bar{I}_{0}+2 \frac{\bar{I}_{1}}{R_{x}}+\frac{\bar{I}_{2}}{R_{x}^{2}}\right), \quad m_{12}=0, \quad m_{13}=\left(\bar{I}_{1}+\frac{\bar{I}_{2}}{R_{x}}\right) \alpha_{\mathrm{m}}, \quad m_{14}=\left(\bar{J}_{1}+\frac{\bar{J}_{2}}{R_{x}}\right) \alpha_{\mathrm{m}}, \\
m_{22}= & -\left(\bar{I}_{0}+\frac{\bar{I}_{1}}{R_{y}}+\frac{\bar{I}_{2}}{R_{y}^{2}}\right), \quad m_{23}=\left(\bar{I}_{1}+\frac{\bar{I}_{2}}{R_{y}}\right) \beta_{n}, \quad m_{24}=\left(\bar{J}_{1}+\frac{\bar{J}_{2}}{R_{y}}\right) \beta_{n}, \\
m_{33}= & -\bar{I}_{0}-\bar{I}_{2}\left(\alpha_{\mathrm{m}}^{2}+\beta_{n}^{2}\right), \quad m_{34}=-\bar{I}_{0}-\bar{J}_{2}\left(\alpha_{\mathrm{m}}^{2}+\beta_{n}^{2}\right), \quad m_{44}=-\bar{I}_{0}-\bar{K}_{1}\left(\alpha_{\mathrm{m}}^{2}+\beta_{n}^{2}\right), \\
k_{11}= & -\left(\bar{A}_{11}+2 \frac{\bar{B}_{11}}{R_{x}}+\frac{\bar{D}_{11}}{R_{x}^{2}}\right) \alpha_{\mathrm{m}}^{2}-\left(\hat{A}_{66}+2 \frac{\hat{B}_{66}}{R_{x}}+\frac{\hat{D}_{66}}{R_{x}^{2}}\right) \beta_{n}^{2}, \\
k_{12}= & -\left(A_{12}+A_{66}+\left(B_{12}+B_{66}\right)\left(\frac{1}{R_{x}}+\frac{1}{R_{y}}\right)+\frac{1}{R_{x} R_{y}}\left(D_{12}+D_{66}\right)\right) \beta_{n}, \\
k_{13}= & \left(\frac{\bar{A}_{11}}{R_{x}}+\frac{A_{12}}{R_{y}}+\frac{1}{R_{x}}\left(\frac{\bar{B}_{11}}{R_{x}}+\frac{B_{12}}{R_{y}}\right)+\left(B_{12}+B_{66}+\hat{B}_{66}+\frac{1}{R_{x}}\left(D_{12}+D_{66}+\hat{D}_{66}\right)\right) \beta_{n}^{2}\right) \alpha_{\mathrm{m}} \\
& +\left(\bar{B}_{11}+\frac{\bar{D}_{11}}{R_{x}}\right) \alpha_{\mathrm{m}}^{3}, \\
k_{14}= & \left(\bar{B}_{11}^{\mathrm{s}}+\frac{\bar{D}_{11}^{\mathrm{s}}}{R_{x}}\right) \alpha_{\mathrm{m}}^{3}+\left(\left(B_{12}^{\mathrm{s}}+B_{66}^{\mathrm{s}}+\hat{B}_{66}^{\mathrm{s}}+\frac{D_{66}^{\mathrm{s}}}{R_{x}}+\frac{\hat{D}_{66}^{\mathrm{s}}}{R_{x}}+\frac{D_{12}^{\mathrm{s}}}{R_{x}}\right) \beta_{n}^{2}\right. \\
& \left.+\frac{\bar{A}_{11}}{R_{x}}+\frac{A_{12}}{R_{y}}+\frac{\bar{B}_{11}}{R_{x}^{2}}+\frac{B_{12}}{R_{x} R_{y}}\right) \alpha_{\mathrm{m}},
\end{aligned}
$$




$$
\begin{aligned}
& k_{15}=-\left(A_{11}^{p}+\frac{B_{11}^{p}}{R_{x}}\right) \alpha_{\mathrm{m}}, \quad k_{16}=-\left(A_{12}^{\mathrm{p}}+\frac{B_{12}^{p}}{R_{x}}\right) \alpha_{\mathrm{m}}, \\
& k_{22}=-\left(\bar{A}_{66}+2 \frac{\bar{B}_{66}}{R_{y}}+\frac{\bar{D}_{66}}{R_{y}^{2}}\right) \alpha_{\mathrm{m}}^{2}-\left(\hat{A}_{22}+2 \frac{\hat{B}_{22}}{R_{y}}+\frac{\hat{D}_{22}}{R_{y}^{2}}\right) \beta_{n}^{2}, \\
& k_{23}=\left(\frac{A_{12}}{R_{x}}+\frac{\hat{A}_{22}}{R_{y}}+\frac{1}{R_{y}}\left(\frac{B_{12}}{R_{x}}+\frac{\hat{B}_{22}}{R_{y}}\right)\right) \beta_{n}+\left(\hat{B}_{22}+\frac{\hat{D}_{22}}{R_{y}}\right) \beta_{n}^{3} \\
& +\left(B_{12}+B_{66}+\bar{B}_{66}+\frac{1}{R_{y}}\left(D_{12}+D_{66}+\bar{D}_{66}\right)\right) \beta_{n} \alpha_{\mathrm{m}}^{2}, \\
& k_{24}=\left(\frac{A_{12}}{R_{x}}+\frac{\hat{A}_{22}}{R_{y}}+\frac{1}{R_{y}}\left(\frac{B_{12}}{R_{x}}+\frac{\hat{B}_{22}}{R_{y}}\right)\right) \beta_{n}+\left(\hat{B}_{22}^{\mathrm{s}}+\frac{\hat{D}_{22}^{\mathrm{s}}}{R_{y}}\right) \beta_{n}^{3} \\
& +\left(B_{12}^{\mathrm{s}}+B_{66}^{\mathrm{s}}+\bar{B}_{66}^{\mathrm{s}}+\frac{1}{R_{y}}\left(D_{12}^{\mathrm{s}}+D_{66}^{\mathrm{s}}+\bar{D}_{66}^{\mathrm{s}}\right)\right) \beta_{n} \alpha_{\mathrm{m}}^{2}, \\
& k_{25}=-\left(A_{21}^{p}+\frac{B_{21}^{\mathrm{p}}}{R_{y}}\right) \beta_{n}, \quad k_{26}=-\left(A_{22}^{\mathrm{p}}+\frac{B_{22}^{p}}{R_{y}}\right) \beta_{n}, \\
& k_{33}=-\frac{\bar{A}_{11}}{R_{x}^{2}}-2 \frac{A_{12}}{R_{x} R_{y}}-\frac{\hat{A}_{22}}{R_{y}^{2}}-2\left(\frac{B_{12}}{R_{x}}+\frac{\hat{B}_{22}}{R_{y}}\right) \beta_{n}^{2}-2\left(\frac{\bar{B}_{11}}{R_{x}}+\frac{B_{12}}{R_{y}}\right) \alpha_{\mathrm{m}}^{2} \\
& -\bar{D}_{11} \alpha_{\mathrm{m}}^{4}-\left(2 D_{12}+2 D_{66}+\bar{D}_{66}+\hat{D}_{66}\right) \alpha_{\mathrm{m}}^{2} \beta_{n}^{2}-\hat{D}_{22} \beta_{n}^{4} \\
& k_{34}=-\frac{\bar{A}_{11}}{R_{x}^{2}}-2 \frac{A_{12}}{R_{x} R_{y}}-\frac{\hat{A}_{22}}{R_{y}^{2}}-\left(\frac{\bar{B}_{11}}{R_{x}}+\frac{\bar{B}_{11}^{\mathrm{s}}}{R_{x}}+\frac{B_{12}}{R_{y}}+\frac{B_{12}^{\mathrm{s}}}{R_{y}}\right) \alpha_{\mathrm{m}}^{2}-\left(\frac{B_{12}}{R_{x}}+\frac{B_{12}^{\mathrm{s}}}{R_{x}}+\frac{\hat{B}_{22}}{R_{y}}+\frac{\hat{B}_{22}^{\mathrm{s}}}{R_{y}}\right) \beta_{n}^{2} \\
& -\left(2 D_{12}^{\mathrm{s}}+2 D_{66}^{\mathrm{s}}+\bar{D}_{66}^{\mathrm{s}}+\hat{D}_{66}^{\mathrm{s}}\right) \alpha_{\mathrm{m}}^{2} \beta_{n}^{2}-\bar{D}_{11}^{\mathrm{s}} \alpha_{\mathrm{m}}^{4}-\hat{D}_{22}^{\mathrm{s}} \beta_{n}^{4}, \\
& k_{35}=B_{21}^{p} \beta_{n}^{2}+B_{11}^{p} \alpha_{\mathrm{m}}^{2}+\frac{A_{21}^{p}}{R_{y}}+\frac{A_{11}^{p}}{R_{x}}, \quad k_{36}=\frac{A_{12}^{p}}{R_{x}}+\frac{A_{22}^{\mathrm{p}}}{R_{y}}+B_{12}^{p} \alpha_{\mathrm{m}}^{2}+B_{22}^{p} \beta_{\mathrm{m}}^{2}, \\
& k_{44}=-\frac{\bar{A}_{11}}{R_{x}^{2}}-2 \frac{A_{12}}{R_{x} R_{y}}-\frac{\hat{A}_{22}}{R_{y}^{2}}-\left(\hat{A}_{44}^{\mathrm{s}}+2 \frac{B_{12}^{\mathrm{s}}}{R_{x}}+2 \frac{\hat{B}_{22}^{\mathrm{s}}}{R_{y}}\right) \beta_{n}^{2}-\left(\bar{A}_{55}^{\mathrm{s}}+2 \frac{\bar{B}_{11}^{\mathrm{s}}}{R_{x}}+2 \frac{B_{12}^{\mathrm{s}}}{R_{y}}\right) \alpha_{\mathrm{m}}^{2} \\
& -\left(2 E_{12}^{\mathrm{s}}+2 E_{66}^{\mathrm{s}}+\bar{E}_{66}^{\mathrm{s}}+\hat{E}_{66}^{\mathrm{s}}\right) \alpha_{\mathrm{m}}^{2} \beta_{n}^{2}-\bar{E}_{11}^{\mathrm{s}} \alpha_{\mathrm{m}}^{4}-\hat{E}_{22}^{\mathrm{s}} \beta_{n}^{4}, \\
& k_{45}=\frac{A_{21}^{p}}{R_{y}}+\frac{A_{11}^{\mathrm{p}}}{R_{x}}+\left(D_{21}^{p}+C_{11}^{p}\right) \alpha_{\mathrm{m}}^{2}+\left(C_{21}^{p}+D_{11}^{p}\right) \beta_{\mathrm{m}}^{2}, \\
& k_{46}=\frac{A_{12}^{p}}{R_{x}}+\frac{A_{22}^{\mathrm{p}}}{R_{y}}+\left(C_{12}^{p}+D_{22}^{p}\right) \alpha_{\mathrm{m}}^{2}+\left(D_{12}^{p}+C_{22}^{p}\right) \beta_{n}^{2}, \\
& k_{55}=-D_{x 12}^{p} \alpha_{\mathrm{m}}^{2}-D_{y 12}^{p} \beta_{\mathrm{m}}^{2}+Q^{p \mathrm{~T}}, \quad k_{56}=0, \quad k_{66}=-D_{x 22}^{p} \alpha_{\mathrm{m}}^{2}-D_{y 22}^{p} \beta_{\mathrm{m}}^{2}+Q^{p \mathrm{~B}} .
\end{aligned}
$$

\section{Appendix B2}

$$
\begin{aligned}
& m_{11}=-\left(\bar{I}_{0}+2 \frac{\bar{I}_{1}}{R_{x}}+\frac{\bar{I}_{2}}{R_{x}^{2}}\right), \quad m_{12}=m_{13}=m_{14}=0, \quad m_{22}=-\left(\bar{I}_{0}+\frac{\bar{I}_{1}}{R_{y}}+\frac{\bar{I}_{2}}{R_{y}^{2}}\right), \\
& m_{23}=m_{24}=0, \quad m_{33}=-\bar{I}_{0}-\bar{I}_{2}\left(\alpha_{\mathrm{m}}^{2}+\beta_{n}^{2}\right), \quad m_{34}=-\bar{I}_{0}-\bar{J}_{2}\left(\alpha_{\mathrm{m}}^{2}+\beta_{n}^{2}\right), \quad m_{44}=-\bar{I}_{0}-\bar{K}_{1}\left(\alpha_{\mathrm{m}}^{2}+\beta_{n}^{2}\right), \\
& k_{11}=-\left(\bar{A}_{11}+2 \frac{\bar{B}_{11}}{R_{x}}+\frac{\bar{D}_{11}}{R_{x}^{2}}\right) \alpha_{\mathrm{m}}^{2}+\left(-2 \frac{\hat{B}_{66}}{R_{x}}-\frac{\hat{D}_{66}}{R_{x}^{2}}-\hat{A}_{66}\right) \beta_{n}^{2}, \\
& k_{12}=-\left(A_{12}+A_{66}+\left(B_{12}+B_{66}\right)\left(\frac{1}{R_{x}}+\frac{1}{R_{y}}\right)+\frac{1}{R_{x} R_{y}}\left(D_{12}+D_{66}\right)\right) \beta_{n} \alpha_{\mathrm{m}}, \\
& k_{13}=\left(\frac{A_{16}}{R_{x}}+\frac{\hat{A}_{16}}{R_{y}}+\frac{1}{R_{x}}\left(\frac{B_{16}}{R_{x}}+\frac{\hat{B}_{26}}{R_{y}}\right)+\left(2 B_{16}+\bar{B}_{16}+2 \frac{D_{16}}{R_{x}}+\frac{\bar{D}_{16}}{R_{x}}\right) \beta_{n} \alpha_{\mathrm{m}}^{2}\right) \beta_{n}+\left(\hat{B}_{26}+\frac{\hat{D}_{26}}{R_{x}}\right) \beta_{n}^{3}, \\
& k_{14}=\left(\frac{\hat{D}_{26}^{\mathrm{s}}}{R_{x}}+\hat{B}_{26}^{\mathrm{s}}\right) \beta_{n}^{3}+\left(\left(2 B_{16}^{\mathrm{s}}+\bar{B}_{16}^{\mathrm{s}}+\frac{\bar{D}_{16}^{\mathrm{s}}}{R_{x}}+2 \frac{D_{16}^{\mathrm{s}}}{R_{x}}\right) \alpha_{\mathrm{m}}^{2}+\frac{A_{16}}{R_{x}}+\frac{\hat{A}_{26}}{R_{y}}+\frac{B_{16}}{R_{x}^{2}}+\frac{\hat{B}_{26}}{R_{x} R_{y}}\right) \beta_{n}, \\
& k_{15}=0, \quad k_{16}=0, \quad k_{22}=-\left(\bar{A}_{66}+2 \frac{\bar{B}_{66}}{R_{y}}+\frac{\bar{D}_{66}}{R_{y}^{2}}\right) \alpha_{\mathrm{m}}^{2}-\left(\hat{A}_{22}+2 \frac{\hat{B}_{22}}{R_{y}}+\frac{\hat{D}_{22}}{R_{y}^{2}}\right) \beta_{n}^{2},
\end{aligned}
$$




$$
\begin{aligned}
& k_{23}=\left(\frac{\bar{A}_{16}}{R_{x}}+\frac{A_{26}}{R_{y}}+\frac{1}{R_{y}}\left(\frac{B_{26}}{R_{y}}+\frac{\bar{B}_{16}}{R_{x}}\right)\right) \alpha_{\mathrm{m}}+\left(\frac{\bar{D}_{16}}{R_{y}}+\bar{B}_{16}\right) \alpha_{\mathrm{m}}^{3}+\left(2 B_{26}+\hat{B}_{26}+\frac{\hat{D}_{26}}{R_{y}}+2 \frac{D_{26}}{R_{y}}\right) \beta_{n}^{2} \alpha_{\mathrm{m}}, \\
& k_{24}=\left(\frac{\bar{A}_{16}}{R_{x}}+\frac{A_{26}}{R_{y}}+\frac{1}{R_{y}}\left(\frac{B_{26}}{R_{y}}+\frac{\bar{B}_{16}}{R_{x}}\right)\right) \alpha_{\mathrm{m}}+\left(\frac{\bar{D}_{16}^{\mathrm{s}}}{R_{y}}+\bar{B}_{16}^{\mathrm{s}}\right) \alpha_{\mathrm{m}}^{3}+\left(2 B_{26}^{\mathrm{s}}+\hat{B}_{26}^{\mathrm{s}}+\frac{1}{R_{y}}\left(D_{26}^{\mathrm{s}}+\hat{D}_{26}^{\mathrm{s}}\right)\right) \alpha_{\mathrm{m}} \beta_{n}^{2}, \\
& k_{25}=0, \quad k_{26}=0 \text {, } \\
& k_{33}=-\frac{\bar{A}_{11}}{R_{x}^{2}}-2 \frac{A_{12}}{R_{x} R_{y}}-\frac{\hat{A}_{22}}{R_{y}^{2}}-2\left(\frac{B_{12}}{R_{x}}+\frac{\hat{B}_{22}}{R_{y}}\right) \beta_{n}^{2}-2\left(\frac{\bar{B}_{11}}{R_{x}}+\frac{B_{12}}{R_{y}}\right) \alpha_{\mathrm{m}}^{2} \\
& -\bar{D}_{11} \alpha_{\mathrm{m}}^{4}-\left(2 D_{12}+2 D_{66}+\bar{D}_{66}+\hat{D}_{66}\right) \alpha_{\mathrm{m}}^{2} \beta_{n}^{2}-\hat{D}_{22} \beta_{n}^{4}, \\
& k_{34}=-\frac{\bar{A}_{11}}{R_{x}^{2}}-2 \frac{A_{12}}{R_{x} R_{y}}-\frac{\hat{A}_{22}}{R_{y}^{2}}-\left(\frac{\bar{B}_{11}}{R_{x}}+\frac{\bar{B}_{11}^{\mathrm{s}}}{R_{x}}+\frac{B_{12}}{R_{y}}+\frac{B_{12}^{\mathrm{s}}}{R_{y}}\right) \alpha_{\mathrm{m}}^{2} \\
& -\left(\frac{B_{12}}{R_{x}}+\frac{B_{12}^{\mathrm{s}}}{R_{x}}+\frac{\hat{B}_{22}}{R_{y}}+\frac{\hat{B}_{22}^{\mathrm{s}}}{R_{y}}\right) \beta_{n}^{2}-\left(2 D_{12}^{\mathrm{s}}+2 D_{66}^{\mathrm{s}}+\bar{D}_{66}^{\mathrm{s}}+\hat{D}_{66}^{\mathrm{s}}\right) \alpha_{\mathrm{m}}^{2} \beta_{n}^{2}-\bar{D}_{11}^{\mathrm{s}} \alpha_{\mathrm{m}}^{4}-\hat{D}_{22}^{\mathrm{s}} \beta_{n}^{4}, \\
& k_{35}=B_{21}^{p} \beta_{n}^{2}+B_{11}^{p} \alpha_{\mathrm{m}}^{2}+\frac{A_{21}^{p}}{R_{y}}+\frac{A_{11}^{p}}{R_{x}}, \quad k_{36}=\frac{A_{12}^{p}}{R_{x}}+\frac{A_{22}^{p}}{R_{y}}+B_{12}^{p} \alpha_{\mathrm{m}}^{2}+B_{22}^{p} \beta_{\mathrm{m}}^{2}, \\
& k_{44}=-\frac{\bar{A}_{11}}{R_{x}^{2}}-2 \frac{A_{12}}{R_{x} R_{y}}-\frac{\hat{A}_{22}}{R_{y}^{2}}-\left(\hat{A}_{44}^{\mathrm{s}}+2 \frac{B_{12}^{\mathrm{s}}}{R_{x}}+2 \frac{\hat{B}_{22}^{\mathrm{s}}}{R_{y}}\right) \beta_{n}^{2}-\left(\bar{A}_{55}^{\mathrm{s}}+2 \frac{\bar{B}_{11}^{\mathrm{s}}}{R_{x}}+2 \frac{B_{12}^{\mathrm{s}}}{R_{y}}\right) \alpha_{\mathrm{m}}^{2} \\
& -\left(2 E_{12}^{\mathrm{s}}+2 E_{66}^{\mathrm{s}}+\bar{E}_{66}^{\mathrm{s}}+\hat{E}_{66}^{\mathrm{s}}\right) \alpha_{\mathrm{m}}^{2} \beta_{n}^{2}-\bar{E}_{11}^{\mathrm{s}} \alpha_{\mathrm{m}}^{4}-\hat{E}_{22}^{\mathrm{s}} \beta_{n}^{4}, \\
& k_{45}=\frac{A_{21}^{p}}{R_{y}}+\frac{A_{11}^{\mathrm{p}}}{R_{x}}+\left(D_{21}^{p}+C_{11}^{p}\right) \alpha_{\mathrm{m}}^{2}+\left(C_{21}^{p}+D_{11}^{p}\right) \beta_{\mathrm{m}}^{2}, \\
& k_{46}=\frac{A_{12}^{p}}{R_{x}}+\frac{A_{22}^{\mathrm{p}}}{R_{y}}+\left(C_{12}^{p}+D_{22}^{p}\right) \alpha_{\mathrm{m}}^{2}+\left(D_{12}^{h p}+C_{22}^{p}\right) \beta_{n}^{2}, \\
& k_{55}=-D_{x 12}^{p} \alpha_{\mathrm{m}}^{2}-D_{y 12}^{p} \beta_{\mathrm{m}}^{2}+Q^{p \mathrm{~T}}, \quad k_{56}=0, \quad k_{66}=-D_{x 22}^{p} \alpha_{\mathrm{m}}^{2}-D_{y 22}^{p} \beta_{\mathrm{m}}^{2}+Q^{p \mathrm{~B}},
\end{aligned}
$$

where

$$
\begin{aligned}
& A_{11}^{p}=\int_{\frac{h_{\mathrm{c}}}{2}}^{\frac{h_{\mathrm{c}}}{2}+h_{\mathrm{p}}}\left(1+\frac{z}{R_{y}}\right) \bar{e}_{31} \frac{-\partial Z_{\mathrm{p}}^{\mathrm{t}}}{\partial z} \phi^{\mathrm{t}} \mathrm{d} z, \quad A_{12}^{p}=\int_{-\frac{h_{\mathrm{c}}}{2}-h_{\mathrm{p}}}^{-\frac{h_{\mathrm{c}}}{2}}\left(1+\frac{z}{R_{y}}\right) \bar{e}_{31} \frac{-\partial Z_{\mathrm{p}}^{\mathrm{d}}}{\partial z} \phi^{\mathrm{d}} \mathrm{d} z, \\
& A_{21}^{p}=\int_{\frac{h_{\mathrm{c}}}{2}}^{\frac{h_{\mathrm{c}}}{2}+h_{\mathrm{p}}}\left(1+\frac{z}{R_{x}}\right) \bar{e}_{32} \frac{-\partial Z_{\mathrm{p}}^{\mathrm{t}}}{\partial z} \phi^{\mathrm{t}} \mathrm{d} z, \quad A_{22}^{p}=\int_{-\frac{h_{\mathrm{c}}}{2}-h_{\mathrm{p}}}^{-\frac{h_{\mathrm{c}}}{2}}\left(1+\frac{z}{R_{x}}\right) \bar{e}_{32} \frac{-\partial Z_{\mathrm{p}}^{\mathrm{d}}}{\partial z} \phi^{\mathrm{d}} \mathrm{d} z, \\
& B_{11}^{p}=\int_{\frac{h_{\mathrm{c}}}{2}}^{\frac{h_{\mathrm{c}}}{2}+h_{\mathrm{p}}}\left(1+\frac{z}{R_{y}}\right) \bar{e}_{31} \frac{-\partial Z_{\mathrm{p}}^{\mathrm{t}}}{\partial z} \phi^{\mathrm{t}} z \mathrm{~d} z, \quad B_{12}^{p}=\int_{-\frac{h_{\mathrm{c}}}{2}-h_{\mathrm{p}}}^{-\frac{h_{\mathrm{c}}}{2}}\left(1+\frac{z}{R_{y}}\right) \bar{e}_{31} \frac{-\partial Z_{\mathrm{p}}^{\mathrm{d}}}{\partial z} \phi^{\mathrm{d}} z \mathrm{~d} z \\
& B_{21}^{p}=\int_{\frac{h_{\mathrm{c}}}{2}}^{\frac{h_{\mathrm{c}}}{2}+h_{\mathrm{p}}}\left(1+\frac{z}{R_{x}}\right) \bar{e}_{32} \frac{-\partial Z_{\mathrm{p}}^{\mathrm{t}}}{\partial z} \phi^{\mathrm{t}} z \mathrm{~d} z, \quad B_{22}^{p}=\int_{-\frac{h_{\mathrm{c}}}{2}-h_{\mathrm{p}}}^{-\frac{h_{\mathrm{c}}}{2}}\left(1+\frac{z}{R_{x}}\right) \bar{e}_{32} \frac{-\partial Z_{\mathrm{p}}^{\mathrm{d}}}{\partial z} \phi^{\mathrm{d}} z \mathrm{~d} z, \\
& C_{11}^{p}=\int_{\frac{h_{\mathrm{c}}}{2}}^{\frac{h_{\mathrm{c}}}{2}+h_{\mathrm{p}}}\left(1+\frac{z}{R_{y}}\right) \bar{e}_{31} \frac{-\partial Z_{\mathrm{p}}^{\mathrm{t}}}{\partial z} \phi^{\mathrm{t}} z^{2} \mathrm{~d} z, \quad C_{12}^{p}=\int_{-\frac{h_{\mathrm{c}}}{2}-h_{\mathrm{p}}}^{-\frac{h_{\mathrm{c}}}{2}}\left(1+\frac{z}{R_{y}}\right) \bar{e}_{31} \frac{-\partial Z_{\mathrm{p}}^{\mathrm{d}}}{\partial z} \phi^{\mathrm{d}} z^{2} \mathrm{~d} z \\
& C_{21}^{p}=\int_{\frac{h_{\mathrm{c}}}{2}}^{\frac{h_{\mathrm{c}}}{2}+h_{\mathrm{p}}}\left(1+\frac{z}{R_{x}}\right) \bar{e}_{32} \frac{-\partial Z_{\mathrm{p}}^{\mathrm{t}}}{\partial z} \phi^{\mathrm{t}} z^{2} \mathrm{~d} z, \quad C_{22}^{p}=\int_{-\frac{h_{\mathrm{c}}}{2}-h_{\mathrm{p}}}^{-\frac{h_{\mathrm{c}}}{2}}\left(1+\frac{z}{R_{x}}\right) \bar{e}_{32} \frac{-\partial Z_{\mathrm{p}}^{\mathrm{d}}}{\partial z} \phi^{\mathrm{d}} z^{2} \mathrm{~d} z \\
& D_{11}^{p}=\int_{\frac{h_{\mathrm{c}}}{2}}^{\frac{h_{\mathrm{c}}}{2}+h_{\mathrm{p}}}\left(1+\frac{z}{R_{x}}\right) g(z) e_{24} \frac{-1}{\left(1+\frac{z}{R_{y}}\right)} Z_{\mathrm{p}}^{\mathrm{t}} \mathrm{d} z, \quad D_{12}^{p}=\int_{-\frac{h_{\mathrm{c}}}{2}-h_{\mathrm{p}}}^{-\frac{h_{\mathrm{c}}}{2}}\left(1+\frac{z}{R_{x}}\right) g(z) e_{24} \frac{-1}{\left(1+\frac{z}{R_{y}}\right)} Z_{\mathrm{p}}^{\mathrm{d}} \mathrm{d} z, \\
& D_{21}^{p}=\int_{\frac{h_{\mathrm{c}}}{2}}^{\frac{h_{\mathrm{c}}}{2}+h_{\mathrm{p}}}\left(1+\frac{z}{R_{y}}\right) g(z) e_{15} \frac{-1}{\left(1+\frac{z}{R_{x}}\right)} Z_{\mathrm{p}}^{\mathrm{t}} \mathrm{d} z, \quad D_{22}^{p}=\int_{-\frac{h_{\mathrm{c}}}{2}-h_{\mathrm{p}}}^{-\frac{h_{\mathrm{c}}}{2}}\left(1+\frac{z}{R_{y}}\right) g(z) e_{15} \frac{-1}{\left(1+\frac{z}{R_{x}}\right)} Z_{\mathrm{p}}^{\mathrm{d}} \mathrm{d} z .
\end{aligned}
$$

